\title{
The Halo Beaming Model for Gamma-Ray Bursts
}

\author{
R.C. Duncan ${ }^{1} \&$ Hui $\mathrm{Li}^{2}$
}

Received —

\footnotetext{
${ }^{1}$ Dept. of Astronomy and McDonald Observatory, Univ. of Texas, Austin, TX 78712; duncan@astro.as.utexas.edu

${ }^{2}$ MS D436, Los Alamos National Laboratory, Los Alamos, NM 87545; hli@lanl.gov
} 


\begin{abstract}
We consider a model for gamma-ray bursts (GRBs) from high-velocity neutron stars in the galactic halo. In this model, bursters are born in the galactic disk with large recoil velocities $\mathbf{V}_{\mathbf{r}}$, and GRBs are beamed to within emission cones of half-angle $\phi_{b}$ centered on $\mathbf{V}_{\mathbf{r}}$. We describe scenarios for magnetically-channeled GRBs that have such beaming characteristics. We then make detailed comparisons of this halo beaming model (HBM) to data from the 3rd BATSE Catalog and from the Pioneer Venus Orbiter experiment, for both GRB intensity and angular position distributions. Acceptable fits to observations of over 1000 bursts are obtained for $\phi_{b}=15^{\circ}-30^{\circ}$ and for a BATSE sampling depth of $D \sim 180 \mathrm{kpc}$, which corresponds to a peak burst luminosity of $\sim 10^{40} \mathrm{ergs} \mathrm{s}^{-1}$. Present data favor a truly isotropic (cosmological) model over the HBM, but not by a statistically compelling margin $(\lesssim 2 \sigma)$.

The HBM makes the distinctive prediction that the galactocentric quadrupole moment $\left\langle\cos ^{2} \Theta\right\rangle-1 / 3$ for bright, nearby GRBs is large, even though the dipole moment $\langle\cos \Theta\rangle$ remains near zero. Bursters born in nearby external galaxies, such as M31, are almost entirely undetectable in the HBM because of misdirected beaming. We analyze several refinements of the basic HBM: gamma-ray intensities that vary with angle from the beam axis; non-standardcandle GRB luminosity functions; and models including a subset of bursters that do not escape from the galaxy. We also discuss the energy budgets for the bursters, the origins of their recoils, and the physics of burst beaming and alignment. One possible physical model is based on the magnetar model of soft gamma repeaters (SGRs). Empirical bounds on the rate of formation and peculiar velocities of SGRs imply that there exist $\sim 10^{4}$ to $\sim 10^{7}$ aged SGRs in the galactic halo within a distance of $100 \mathrm{kpc}$.
\end{abstract}


The HBM gives an acceptable fit to observations only if it satisfies some special conditions ( $\phi_{b} \approx 20^{\circ}$, uniform bursting rate) which are possible, but for which there are no clear and compelling theoretical justifications. The cosmological burst hypothesis is more generic and thus more attractive in this sense.

Subject headings: gamma rays: bursts — stars: magnetic fields — stars: neutron - galaxy: halo 


\section{Introduction}

The Burst and Transient Source Experiment (BATSE) on the NASA Compton GammaRay Observatory has revealed a nearly isotropic but inhomogeneous sky distribution of gamma-ray bursts (Meegan et al. 1992; Meegan et al. 1996). An additional major constraint on GRB theories, revealed by the Pioneer Venus Orbiter (PVO) experiment, is that the intensity distribution of bright bursts is consistent with a locally-uniform density of sources in Euclidean space (Fenimore et al. 1993). These facts are simply and generically accounted for if the faintest observed GRBs come from cosmological distances (e.g., Paczyński 1995). An alternative possibility, that we will focus on here, is that the bursts come from the extended halo or corona of our galaxy. Galactic halo models for GRBs were first considered and tested against data by Fishman et al. (1978) and Jennings \& White (1980). Reasons for favoring halo models have been summarized by Lamb (1995).

In galactic halo GRB models, it is often supposed that the bursters are neutron stars, since such compact stars are conjectured to be capable of producing intense fluxes of hard, non-thermal photons; while their small size could drive burst variability on submillisecond time scales. Other reasons for favoring neutron stars include observations of spectral lines, which are at present controversial, and a possible connection of classic GRBs with the March 5, 1979 gamma ray burster (e.g., Duncan \& Thompson 1992, hereafter DT92) which was localized to an angular position lying within a young supernova remnant (Cline et al. 1982; Rothschild, Kulkarni \& Lingenfelter 1994). The displacement of this burster from the center of the supernova remnant indicates that it acquired a velocity $\sim 1000 \mathrm{~km} \mathrm{~s}^{-1}$ at birth (DT92). Neutron stars with such velocities will escape the galactic disk, and move into the halo. Recent analysis of the 1979 March 5th event lends support to its association with classical GRBs (Fenimore, Klebesadel, \& Laros 1996, hereafter FKL). In addition, many high-velocity radio pulsars $\left(V>500 \mathrm{~km} \mathrm{~s}^{-1}\right)$ have been observed (Lyne \& Lorimer 
1994; Frail 1996).

For these reasons, we will focus on theories of GRBs from high-velocity neutron stars (HVNSs) born in the galactic disk, as first suggested by Shklovskii \& Mitrofanov (1985). In particular, we will consider the "halo beaming model" (HBM) proposed earlier (Duncan, Li, \& Thompson 1993, hereafter DLT; Li, Duncan, \& Thompson 1994, LDT; Li \& Duncan, 1996a, 1996b; Bulik \& Lamb 1996). Alternative models for GRBs from HVNSs in the galactic halo invoke a delayed onset of bursting activity at a time $\sim 10^{7}$ years after birth in the disk (Li \& Dermer 1992, hereafter LD92), which has been applied in several different physical contexts (Colgate \& Leonard 1994; Lamb, Bulik \& Coppi 1996; Woosley \& Herant 1996); and weakly-bound bursters orbiting in a nonspherical galactic potential (Podsiadlowski, Rees \& Ruderman 1995, hereafter PRR). Models for bursters born in the galactic halo (Eichler \& Silk 1992; Hartmann 1992; Salpeter \& Wasserman 1993; Wasserman \& Salpeter 1994), or the Magellanic Clouds (Fabian \& Posiadlowski 1993), are beyond the scope of this paper.

In $\S 2$, we will describe our basic model for the galactic population of bursters, and briefly review its physical motivations. In $\$ 3$ we present our basic Monte Carlo model results. We discuss how these results arise, and what they could imply for halo GRB theories. We also explore the model's sensitivity to different beaming angles. In §四 we make detailed quantitative comparisons of the model with BATSE and PVO data. In particular, we study moments of the angular position distribution in bright subsets of observed GRBs, which is potentially a sensitive model discriminant. In $\S 5$ we estimate the GRB repetition rate and the energy requirements for bursters. We also discuss two candidate power sources that could satisfy these requirements: magnetic energy and accretion energy. Several refinements of the basic HBM are investigated in $\S 6$, namely: gamma-ray intensities that vary with angular position within the beam, GRB luminosity functions, and models with 
a significant subset of bursters on bound orbits in the galactic halo. In $\$$ ] we give our conclusions and outline future observational tests.

Note that we consider possible physical mechanisms for halo GRBs, involving neutron stars with unusually strong magnetic fields, in $\S 2.3, \S 2.4, \S 5, \S 6.3$, and Appendix B. These sections could be read separately from the rest of the paper, since they might apply in a non-beamed model context (cf. $\S 7.2)$.

\section{The Halo Beaming Model: Physical Motivations}

\subsection{Model Assumptions}

We will assume that GRBs are emitted by HVNSs, which emanate from the galactic disk like a "wind" extending into the galactic corona (Shklovskii \& Mitrofanov 1985). The HBM does not require that the bursters or their peculiar velocities have any favored orientation in a galactic coordinate frame, but it does invoke the physically-plausible condition that gamma-rays are produced only within a cone of angular radius $\phi_{b}$ about the star's magnetic axis $\pm \vec{\mu}$. The magnetic axis is furthermore assumed to be roughly aligned (within $\sim 20^{\circ}$ ) with the stellar recoil velocity $\mathbf{V}_{\mathbf{r}}$. We discuss the physics of such beaming and alignment in $\S 2.2$.

The particular version of HBM which we will analyze quantitatively in $\S 3$ and $\S$ 团 below has the following simple properties: [1] bursters are born at positions distributed like young Pop. I stars in the galactic disk; [2] with randomly directed recoils $V_{r}=1000 \mathrm{~km} \mathrm{~s}^{-1}$; [3] they emit GRBs at a constant rate, with [4] constant luminosity, and [5] the gamma ray emission is beamed parallel and anti-parallel to $\mathbf{V}_{\mathbf{r}}$, within an angular radius $\phi_{b}$ that we will vary.

GRB beaming in a direction correlated with $\mathbf{V}_{\mathbf{r}}$ makes the observable burst distribution 
comply with the BATSE dipole isotropy and the PVO brightness-distribution constraints, for the following reasons (see Figure [1). Since the HVNSs are freely-streaming out of the galaxy, their mean density diminishes with distance from the galactic center as $n \sim r^{-2}$. However, the fraction of escaping bursters that are potentially detectable at Earth increases in proportion to the transverse area of their beaming cones, $\sim r^{2}$. These two trends cancell, making the effective number of bursters increase linearly with the sampled volume (i.e., an apparent "constant density" of detectable stars) within a "core radius" $R_{c} \sim R_{o} / \phi_{b}$, where $R_{o} \sim 8.5 \mathrm{kpc}$ is a galactic disk dimension. In the HBM, this produces the the observed "homogeneous" distribution of bright GRBs found by the PVO experiment. Furthermore, since most bursters are undetectable when they are at small $r$, the observable dipole anisotropy of GRB positions in the direction of the galactic center is greatly reduced. At distances larger than $R_{c}$, all bursters are detectable at Earth (or nearly all; see exceptions discussed in $\S 3$ ), and the $n \propto r^{-2}$ free-streaming fall-off of burster density prevails, accounting for the "boundedness" $\left(\left\langle V / V_{\max }\right\rangle<0.5\right)$ found by BATSE.

A more detailed illustration of the geometrical effect of burst beaming is given in Figure 2. To understand this figure, consider for a moment an idealized model in which all stars are born precisely at the galactic center (GC), and move out in random directions on straight-line trajectories, each emitting GRBs into a cone of half-opening angle $\phi_{b}$ around its velocity vector. Bursters lying outside the circles in Figure 2, or within the lens-shaped intersection of circles between the GC and Earth, are then the only ones that can be detected at Earth. There is a large "zone of avoidance" (ZOA), within which all bursters are invisible. Figure 2 actually shows only the 2-D cross-section of this ZOA. The true $\mathrm{ZOA}$ is the volume of revolution of the pictured shape about the line between Earth and the galactic center. The boundary in Figure 2 at which stars just become observable (edge of the ZOA) is part of a circle with radius $D_{\text {sun }} / \sin \phi_{b}$, where $D_{\text {sun }}=8.5 \mathrm{kpc}$ is the distance from Sun to the galactic center. 
In the realistic situation, bursters are born throughout the galactic disk, with a peak birth rate at $\sim 4-5 \mathrm{kpc}$ from the center, where the greatest concentration of Population I stars are located (van der Kruit 1987). Each birthplace in the disk then has its own ZOA, scaling up or down in linear size with the distance between the birthplace and Earth. One must add the weighted distributions of detectable bursts together to get the total observable GRB distribution. There is no simple, analytic way to do this, so in what follows we will use Monte Carlo methods. We will also calculate realistic trajectories in the galactic potential, rather than assuming straight lines.

\subsection{Physics of Beaming and Alignment}

Gamma-ray bursts from high- $B$ environments tend to be beamed along field lines because of the transverse pair-creation opacity (e.g., Riffert, Mészáros \& Bagoly 1989; Ho, Epstein \& Fenimore 1990) and because gamma rays produced by such mechanisms as curvature radiation and Compton upscattering are strongly beamed along field lines (e.g., Sturrock 1986; Dermer 1990 and references therein). Even locally-isotropic emissions are strongly beamed when they occur in a relativistic outflow channeled by a magnetic field (e.g., Yi 1993). Gamma emissions induced by sheared Alfvén waves in a neutron star magnetosphere can also be highly beamed (Melia \& Fatuzzo 1991; Fatuzzo \& Melia 1993). Beaming obviates the $\gamma-\gamma$ opacity limit for GRBs (Krolik \& Pier 1991; Baring 1993; Fenimore Epstein \& Ho 1993).

The HBM invokes the additional (assumed) property that burster recoil velocities are approximately aligned with magnetic dipole - and hence burst beaming - axi: $\vec{V}_{r} \| \vec{\mu}$.

This requires that the rotation axis $\vec{\Omega}$ is also roughly aligned with $\vec{\mu}$, at least to within the burst opening angle $\phi_{b}$. 
Is $\vec{V}_{r} \| \vec{\mu}$ a plausible assumption? Any recoil mechanism which imparts impulses to the stellar surface with a coherence time longer than the rotation period of the star will tend to make $\vec{V}_{r} \| \pm \vec{\Omega}$ because of "rotational averaging." One such recoil mechanism is anisotropic neutrino emission during the first $\sim 10 \mathrm{~s}$ after formation ( $\S 2.4$ below). If it is also true that $\vec{\mu} \| \pm \vec{\Omega}$ (at least to within the beaming angle $\phi_{b}$ ) then the alignment condition of the HBM is satisfied.

Such near-alignment between $\vec{\mu}$ and $\pm \vec{\Omega}$ is expected if the neutron star magnetic field is generated by large-scale dynamo action, as in familiar stellar and planetary dynamos. The "magnetar" model, described below (§2.3), is one scenario involving such a dynamo. Vacuum magnetic torques increase alignment (Michel \& Goldwire 1970; Davis \& Goldstein 1970) after the star spins down past the "death line" at which magnetospheric currents are quenched, at age $t_{D} \sim 10^{5}\left(B_{\text {dipole }} / 10^{14} \mathrm{G}\right)^{-1}$ yrs (Chen \& Ruderman 1993). Before this point is reached, currents might exert counter-aligning torques of comparable strength (e.g., McKinnon 1993 and references therein).

Note that the GRB beaming angle $\phi_{b}$ is the sum of the intrinsic beaming angle due to gamma-ray opacity and radiative effects, and the r.m.s. scatter in the angle between $\vec{\Omega}$ and $\vec{\mu}$. Gamma production mechanisms such as curvature radiation and Compton upscattering could operate at significant altitudes in the magnetosphere, where field lines are tilted with respect to $\vec{\mu}$, yielding larger values of $\phi_{b}$ than one would estimate under the assumption of near-polar gamma emission (as adopted by Riffert, Mészáros \& Bagoly 1989; Ho, Epstein \& Fenimore 1990). Values as large as $\phi_{b} \sim 20^{\circ}$ are possible. 


\subsection{Magnetically-powered Bursts?}

The HBM might apply in a variety of different physical contexts. Here we will briefly discuss the "magnetar" model for GRBs (DT92). The magnetar idea has previously been invoked in models of soft gamma repeater bursts (Thompson \& Duncan 1995, hereafter TD95) as well as in models of continuous X-ray emissions from SGRs and from anomalous X-ray pulsars like 1E2259+586 (Thompson \& Duncan 1996, TD96).

Magnetars are hypothetical neutron stars which are born with dipole fields in excess of $B_{Q} \equiv m e^{2} c^{3} / e \hbar=4.4 \times 10^{13} \mathrm{G}$. How might such stars form? Neutron stars are hot and convective during the first $\sim 10$ seconds after they form. They also undergo strong differential rotation (TD93). Large-scale dynamo action might operate efficiently during this time for neutron stars with mean rotation periods below a threshold comparable to the convective overturn time, as predicted by $\alpha-\Omega$ dynamo theory. There is evidence for such a threshold in magnetically-active main-sequence stars (e.g., Simon 1990). This threshold effect could give rise to a bimodal population of neutron stars ("pulsars and magnetars") with a factor $\gtrsim 10^{2}$ difference in field strength (DT92). A more detailed explanation of this magnetar formation hypothesis is given by Duncan \& Thompson (1996, DT96). Seven distinct estimates of the field in the bursting neutron star SGR 0526-66 seem to indicate $B>10^{14} \mathrm{G}(\mathrm{TD} 95) \cdot$

\footnotetext{
${ }^{3}$ Katz (1996) has raised some interesting questions about theories of SGR burst emissions. Our views on these points are given in TD95. In particular, equation (2) of TD95 explains why we believe that plasma-confining magnetic fields $\gtrsim 10^{14} \mathrm{G}$ are necessary in SGR 052666; $§ 3.2$ of TD95 explains why we do not favor a model for SGR bursts involving a pure pair plasma confined by a $\sim 10^{12} \mathrm{G}$ field, as favored by Katz; and $\S 7.3 .2$ explains why we do not favor a hydrostatic model in which the magnetic Eddington limit operates without confining magnetic stresses, which Katz incorrectly attributes to us. Another good argument against
} 
Magnetars spin down too rapidly to be observed as radio pulsars. DT92 conjectured that GRBs are flare-like reconnection events in a galactic halo population of such strongly-magnetized neutron stars. We will show in $\S 4.5$ that the available magnetic energy is sufficient to power observed GRBs, in the context of the HBM (see also PRR).

Magnetic reconnection is a theoretically advantageous energy source for GRBs, for several reasons. Gravitational and nuclear energy releases generally occur in bulk baryonic matter, which has many degrees of freedom into which energy can thermalized and degraded via adiabatic expansion (e.g., Piran \& Shemi 1993). Flares in a neutron star magnetosphere, on the other hand, can be "clean," exciting only photon and pair degrees of freedom to a first approximation. Large scale electromotive forces induced by reconnection will generally accelerate pairs, and may produce hard, non-thermal spectra via Compton upscattering, synchrotron emission, and curvature radiation (Sturrock 1986). Indeed, GRBs have many qualitative similarities to stellar flares. Both are transient energy releases, with chaotic variability over a wide range of time scales; and both have spectrally-hard non-thermal components (e.g., Murphy et al. 1993; Ramaty \& Mandzhavidze 1993). The similarities are so strong that a minor fraction of bursts in the BATSE catalog might actually be intense events from nearby, non-neutron, flare stars (Liang \& Li 1993). Stochastic avalanche models give a good fit to GRB time profiles (Stern \& Svensson 1996) and to many properties of solar flares (Lu et al. 1993).

If the $V_{r}-B_{\text {dipole }}$ correlation observed in some samples of radiopulsars (e.g., Cordes 1986; Stollman \& van den Heuvel 1986) is applicable for all neutron stars, then the mean magnetar recoil velocity would be $\gtrsim 10$ times larger than the mean for radiopulsars (but see Lorimer, Lyne and Anderson 1995). Several mechanisms predict unusually large recoils for magnetars, sufficient to propel them into the galactic halo, as explained in $\S 2.4$ below.

hydrostatic models, based on some novel physics, is given by Miller (1995). 
Because of the dynamo origins of magnetar fields, one expects that initially $\vec{\Omega}, \pm \vec{V}_{r}$ and $\pm \vec{\mu}$ are roughly aligned in magnetars. As the star spins down, magnetospheric currents might drive some degree of misalignment; however, once the star spins down past the "death line" at time $\sim 10^{5}\left(B_{\text {dipole }} / 10^{14} \mathrm{G}\right)^{-1}$ yrs (Chen \& Ruderman 1993), further spindown certainly enforces alignment (Michel \& Goldwire 1970; Davis \& Goldstein 1970), since the magnetostatic stellar distortion in magnetars is large enough to damp nutations of $\vec{\Omega}$ about $\vec{\mu}$ (Goldreich 1970, DLT). Incidentally, this is probably not true in old, spun-down $\left(P_{\text {rot }}>4 \mathrm{~s}\right)$ radiopulsars. We conclude that $\vec{V}_{r}$ is likely to be roughly aligned with $\pm \vec{\mu}$ in old magnetars, where $\pm \vec{\mu}$ is also the axis of beamed gamma emissions (DLT). p

This scenario for classic GRBs assumes that magnetars retain strong dipole magnetic fields. Large-scale magnetic instabilities that reduce $B_{\text {dipole }}$ (Flowers \& Ruderman 1979) might be suppressed by toroidal field components in the stellar interior, as expected for fields generated via $\alpha-\Omega$ dynamos, or perhaps by other mechanisms (TD93 $\$ 14.2$ ). Note that a dipole field anchored in the stably-stratified liquid interior of a magnetar cannot be greatly distorted by spindown-induced crustal tectonic drift (Ruderman 1991) because magnetic stresses dominate tensile stresses in the crust. This might differ markedly from the circumstance in ordinary radiopulsars (Ruderman 1991). The interactions of vortex

\footnotetext{
${ }^{4}$ Magnetically-induced neutrino starspots, as discussed in $§ 2.4$, will have coherence times $\tau_{\text {spot }} \gtrsim P_{\text {rot }}$ when the convective overturn time satisfies $\tau_{\text {con }} \gtrsim P_{\text {rot }}$, thus "rotational averaging" implies that $\vec{V}_{r} \| \pm \vec{\Omega}$ (where $\Omega=2 \pi / P_{\text {rot }}$ ); and $\vec{\Omega} \| \pm \vec{\mu}$ because the dipole field is generated by an $\alpha-\Omega$ dynamo.

${ }^{5}$ Fenimore, Klebesadel \& Laros (1996) have suggested that an initial period of (spindowninduced) misalignment occurs during the SGR phase, allowing bursts like the 1979 March 5th event to be observed. Subsequent alignment by vacuum magnetic torques occurs when classic GRB emissions begin.
} 
lines in the neutron superfluid with superconducting flux tubes are also probably irrelevant for young magnetars, because the interior field is probably strong enough to suppress superconductivity. A young magnetar's field evolves predominantly via ambipolar diffusion in the interior and Hall fracturing in the crust (TD96). As the interior field decays, superconductivity will appear in the mantle and perhaps the core; but this probably happens only after rapid spindown has driven most superfluid vortex lines out of the interior. Further discussion of magnetar evolution is given by PRR.

\subsection{Neutrino Magnetic Recoils}

A dipole anisotropy of only $\sim 0.03$ in the neutrino emission from a young, hot neutron star would impart a recoil velocity $\sim 1000 \mathrm{~km} \mathrm{~s}^{-1}$ to the star (Chugai 1984). Here we briefly review mechanisms whereby neutrino anisotropies could be magnetically induced. Purely hydrodynamic (non-magnetic) mechanisms for producing neutron star recoils have been proposed by Janka \& Müller (1994), Shimizu, Yamada \& Sato (1994), Burrows \& Hayes (1996), and in references quoted therein.

A strong magnetic field affects neutrino emissions from the beta processes $n \rightarrow p^{+} e^{-} \nu$ and $p^{+} e^{-} \rightarrow n \bar{\nu}$, as calculated by Dorofeev, Rodionov \& Ternov (1985), and from neutrino scattering processes as calculated by Vilenkin (1995). Dorofeev et al. and Vilenkin furthermore estimated the neutron star recoils resulting from these processes, in the uniform-field idealization. This is a macroscopic manifestion of parity-nonconservation in the weak interactions. Đ

\footnotetext{
${ }^{6}$ Another neutrino magnetic recoil estimate, by Bisnovatyi-Kogan (1996), makes several assumptions: (1) the neutron star magnetic field undergoes simple linear winding driven only by differential rotation (i.e., ignoring all mixing motions); (2) the winded field is
} 
In a realistically non-uniform magnetic field, the back-reaction of magnetic stresses on convective energy flow, along with the neutrino opacity variations outlined above, would give rise to "neutrino starspots," analogous to sunspots, and thus produce neutron star recoils of a magnitude estimated in DT92 and $\S 13$ of TD93.

These calculations are based on standard Weinberg-Salam weak interaction theory. If neutrinos have mass, on the other hand, a strong magnetic field will shift resonant flavor-changing (MSW) oscillations, thereby also inducing an anisotropy in the emergent neutrino flux from a nascent neutron star (Kusenko \& Segrè 1996).

All of these mechanisms produce recoils which vary directly—usually linearly—with the mean magnetic field strength: $V_{r} \propto B$. Thus if any of these mechanisms dominate, one would expect much larger recoils for magnetars than for pulsars. Several non-neutrino recoil mechanisms which also might operate more efficiently in magnetars than in pulsars were discussed by DT92."

To estimate neutrino magnetic recoils realistically, one must know the strength,

largely cancelled by a postulated pre-existing toroidal field in one hemisphere only; (3) the free neutron decay rate in a magnetic field applies. These assumptions are questionable. In particular, realistic estimates of the beta neutrino emissivity within a nascent neutron star should take into account the presence of magnetized, degenerate, relativistic electrons (Dorofeev, Rodionov \& Ternov 1985). Dorofeev et al.'s work was itself an improvement on Chugai's pioneering calculation, which did not treat the energy levels of the electrons in a $B \gg B_{Q}$ field correctly.

${ }^{7} \mathrm{~A}$ star with an oblique, off-center dipole field experiences a spindown-driven recoil (Harrison \& Tadmaru 1975), but this is unlikely to produce velocities as large as $\sim 1000 \mathrm{~km}$ $\mathrm{s}^{-1}$, as explained in DT92. 
coherence length and coherence time of the magnetic field during the epoch of maximum neutrino luminosity. This is not possible at present. However, if the magnetic field approaches equipartition with the free energy of differential rotation and/or the convective fluid mixing — which is strongly in the MHD limit - then $B \gtrsim 10^{16} \mathrm{G}$ at the neutrinosphere (TD93). Such strong fields could be present when most of the neutrino energy is radiated away, even if the surface dipole field, which is frozen-in tens of seconds later by the onset of stable stratification in the liquid interior (Goldreich \& Reisenegger 1992), is smaller by a factor of $\sim 10$ or $\sim 30$. Recoils $V_{r} \gtrsim 10^{3} \mathrm{~km} \mathrm{~s}^{-1}$ are plausible.

\section{Galactic Halo Model Results}

By integrating a large number $\left(\sim 10^{6}\right)$ of neutron star trajectories in a realistic galactic potential, we have derived the sky distribution of HVNSs. In the model described here ( $\S 3$ and $\S 4$ ), all stars have $\left|\mathbf{V}_{\mathbf{r}}\right|=1000 \mathrm{~km} \mathrm{~s}^{-1}$; thus they move in nearly straight lines and eventually escape from the galaxy (but see $§ 6.3$ ).

We used a Monte Carlo code developed by Li \& Dermer (1992), which in turn follows many of the prescriptions of Paczyński (1990) and Hartmann, Epstein \& Woosley (1990). In particular, we adopt van der Kruit's (1987) model for the spatial distribution of young Pop. I stars which spawn neutron stars in the galactic disk. The trajectories of $\sim 10^{6}$ neutron stars were numerically integrated in a realistic galactic potential (Miyamoto \& Nagai 1975) 9 including a dark halo cutoff at radius $R_{H}=70 \mathrm{kpc}$. The escape velocity from

\footnotetext{
${ }^{8}$ Lamb, Bulik, \& Coppi (1996) have argued that the galactic potential of Miyamoto \& Nagai (1975) implies an unrealistically extended disk which tends to 'focus' neutron star orbits into the disk plane. We find, however, that this focusing effect is negligible for randomly oriented initial recoil velocities of magnitude $V_{r} \gtrsim 1000 \mathrm{~km} \mathrm{~s}^{-1}$. This is because the disk is
} 
the center of this model potential is $V_{e s c} \sim 600 \mathrm{~km} \mathrm{~s}^{-1}$. This increases only logarithmically with $R_{H}$, reaching $800 \mathrm{~km} \mathrm{~s}^{-1}$ for $R_{H}=200 \mathrm{kpc}$, thus our results are probably insensitive to $R_{H}$ over its plausible range (LD92). Deviations from sphericity in the dark halo were neglected (but see PRR). We also do not include the potential of M31 since stars with $V_{r} \sim 10^{3} \mathrm{~km} \mathrm{~s}^{-1}$ are negligibly perturbed by M31 within the BATSE sampling depth we found of $<200 \mathrm{kpc}(\S 4.1)$.

In Figure 3 we show HBM numerical results for several angular statistics, plotted as functions of sampling depth $D$ about the Earth. That is, the figure shows cumulative angular statistics for all detectable bursters at distances from Earth that are less than or equal to the value of $D$ on the horizontal axis. The topmost plot of Figure 3 shows the galactocentric dipole moment $\langle\cos \Theta\rangle$, where $\Theta$ is the angle between a burst and the galactic center. The second plot shows the disk-like quadrupole, $\left\langle\sin ^{2} b\right\rangle-1 / 3$, where $b$ is galactic latitude; $\left\langle\sin ^{2} b\right\rangle<1 / 3$ implies that sources are concentrated toward the disk. The third plot shows the galactocentric quadrupole, $\left\langle\cos ^{2} \Theta\right\rangle-1 / 3$. The bottom plot of Figure 3 shows $\left\langle V / V_{\max }\right\rangle$, a statistic which is related to the slope of the cumulative $\log \mathcal{N}-\log P$ brightness distribution, as explained below.

Within each subplot of Figure 3, the various lines correspond to different beaming angles $\phi_{b}$ as described in the figure caption. We have cut off the $\phi_{b}=10^{\circ}$ and $5^{\circ}$ curves for $D \leq 10 \mathrm{kpc}$ because our Monte Carlo sampling statistics at smaller $D$ are too poor. For example, the number of detectable stars at $D \leq 3 \mathrm{kpc}$ in our Monte Carlo model is only $\sim 55$ for $\phi_{b}=5^{\circ}$, whereas it is $\sim 10^{4}$ for in the unbeamed case $\left(\phi_{b}=90^{\circ}\right)$.

Increasing the sampling depth $D$ (moving to the right in Figure 3) is tantamount to including fainter and fainter bursts. Eventually the BATSE sampling depth is reached; at only a very minor part of the total mass of the Galaxy. 
this point, if the model is to fit observations, all the plotted statistics must simultaneously match BATSE values to within observational uncertainty. The most recent published BATSE results are plotted in Figure 3 at a value $D=180 \mathrm{kpc}$. Before discussing these results, we must explain the significance of $\left\langle V / V_{\max }\right\rangle$.

The $V / V_{\max }$ statistic was invented for the study of the quasars (Schmidt 1968), and was first applied to GRBs by Schmidt, Higdon \& Hueter (1988). For scintillation counter experiments, $\left\langle V / V_{\max }\right\rangle$ is the average of $\left(C / C_{\min }\right)^{-3 / 2}$ over all bursts, where $C$ is the peak counts (in a set time interval) and $C_{\min }$ is the threshold for detection. $C_{\min }$ can vary with background noise and other effects (e.g., "overwrites"); however, for a source population that is uniformly distributed in static Euclidean space, $\left\langle V / V_{\max }\right\rangle$ is equal to 0.5 regardless of how the threshold varies; and $\left\langle V / V_{\max }\right\rangle<0.5$ indicates that the density of bursters diminishes with distance, for standard-candle sources. Because $C_{\min }$ is variable, the $\left\langle V / V_{\max }\right\rangle$ statistic is useful in the study of inhomogeneous data sets only when trying to answer the yes/no question: "Is the observed brightness distribution consistent with a uniform density of sources distributed in Euclidean space?" (e.g., Band 1992; Petrosian $1993)$.

In Figure 3, we use $\left\langle V / V_{\max }\right\rangle$ in a purely illustrative way. Our model values of $\left\langle V / V_{\max }\right\rangle$ are ideal values that would be found by an instrument with a uniform detection threshold, i.e., no variations in the noise or the threshold settings. Since the $C_{m i n}$ values in the BATSE catalog are not highly variable (Meegan et al. 1996) preliminary comparisons with BATSE,

\footnotetext{
${ }^{9}$ Results from the third BATSE catalog for 1122 bursts (Meegan et al. 1996) are as follows: $\langle\cos \Theta\rangle_{\text {data }}=-0.002 \pm 0.017$. There is a concurrent anisotropy in the BATSE sky coverage of $\langle\cos \Theta\rangle_{s k y}=-0.013$. Thus the best measure of the burst population dipole moment is $\langle\cos \Theta\rangle=\langle\cos \Theta\rangle_{\text {data }}-\langle\cos \Theta\rangle_{\text {sky }}=+0.011 \pm 0.017$. Similarly, $\left\langle\sin ^{2} b\right\rangle-1 / 3=0.002 \pm 0.009$, and for 657 bursts, $\left\langle V / V_{\max }\right\rangle=0.33 \pm 0.01$.
} 
as in the bottom panel of Figure 3, will not lead us astray. However, the most accurate way to compare burst observations with theory is to fit the observed distribution of peak photon fluxes to Monte Carlo models which have been realistically filtered for detection incompleteness (e.g., Lubin \& Wijers 1993). This is what we do in our actual statistical comparisons of the HBM with the BATSE catalog $(\S 4)$.

Note that the model curves for angular statistics in Figure 3 implicitly assume a detector with uniform sky coverage; i.e., we have idealized that the detector is equally capable of detecting bursts from any location on the celestial sphere. We have corrected for the imperfect sky coverage of BATSE by shifting the data points in Figure 3 appropriately (see footnote 9). In $\S$ we will take the opposite approach: using the raw BATSE data and filtering the Monte Carlo model to take into account BATSE's imperfect sky coverage.

What can be learned from Figure 3]? Beaming evidently increases $\left\langle V / V_{\max }\right\rangle$ to nearly 0.5 for nearby bursters, $D \sim 30 \mathrm{kpc}$. This can make the model satisfy PVO constraints, as we show quantitatively below. Beaming also evidently reduces $\langle\cos \Theta\rangle$. The $\phi_{b}=20^{\circ}$ case fits BATSE observations over an appreciable range of sampling depths $D>100 \mathrm{kpc}$.

Note that the largest deviations from isotropy in Figure 3 occur for bright (i.e., small- $D$ ) subsets of the observable bursts. A distinctive signature of the HBM is that $\left\langle\cos ^{2} \Theta\right\rangle-1 / 3$ is positive for bright bursts. This can be understood as follows. The (bright) bursters which become visible at Earth before reaching the remote galactic halo are the ones which happen to be born with beaming axis $\vec{\mu}$ (and hence, with $\vec{V}_{r}$ ) pointed approximately toward or away from Earth (to within $\sim \phi_{b}$ ). Because most bursters are born within the Solar circle in the galactic disk, the brightest ones tend to be seen in that direction and toward the galactic anticenter, if they have already moved past the Earth on their way out of the galaxy. Thus $\left\langle\cos ^{2} \Theta\right\rangle>1 / 3$ for bright bursts, while the dipole moment $\langle\cos \Theta\rangle$ remains small. 
All burst sources can be seen at distances $D \gg R_{o} / \phi_{b}$ in the $\phi_{b}=\left\{90^{\circ}, 40^{\circ}\right.$ and $\left.20^{\circ}\right\}$ cases of Figure 3, thus in a first approximation, one would expect $\left\langle\sin ^{2} b\right\rangle$ to reach the isotropic value of $1 / 3$ asymptotically at large $D$. In fact, a mild degree of disklike quadrupole anisotropy, $\left\langle\sin ^{2} b\right\rangle=0.31$, remains in these models at large $D$. This is due to the galactic disk rotation (with velocity $V_{\Omega} \approx 220 \mathrm{~km} \mathrm{~s}^{-1}$ ), which makes bursters tend to stream out at lower galactic latitudes, diminishing the density of bursters near the galactic poles compared to the equator at a given distance $r$ from the galactic center (LD92). This effect is not dependent on beaming. It depends only on the ratio $V_{\Omega} / V_{r}$, with $\left\langle\sin ^{2} b\right\rangle \rightarrow 1 / 3$ at large $D$ as $V_{\Omega} / V_{r} \rightarrow 0$.

Galactic rotation also causes the total initial velocity vector $\overrightarrow{V_{r}}+\overrightarrow{V_{\Omega}}$ to be inclined by as much as as

$$
\phi_{V}^{\max } \equiv \arctan \left(V_{\Omega} / V_{r}\right) \approx 12^{\circ}\left(\frac{V_{r}}{1000 \mathrm{~km} \mathrm{~s}^{-1}}\right)^{-1}
$$

with respect to the axis of GRB emission, $\vec{V}_{r} / V_{r}$. Thus if $\phi_{b}<\phi_{V}^{\text {max }}$, some stars never become visible from Earth. Since this happens preferentially at high galactic latitudes, $\left\langle\sin ^{2} b\right\rangle$ is always substantially less than $1 / 3$ in models with $\phi_{b}<\phi_{V}^{\max }$. For example, in the case $\phi_{b}=10^{\circ},\left\langle\sin ^{2} b\right\rangle$ never exceeds 0.25 . Only when $\phi_{b}>\phi_{V}^{\max }$ are all stars visible at large $D$, causing $\left\langle\sin ^{2} b\right\rangle$ to asymptote to the unbeamed, large $-D$ value of 0.31 .

When $\phi_{b}<\phi_{V}^{\max }$ most stars eventually become undetectable at Earth, but only after passing through a phase when they can be seen, namely, when $D<8.5 \mathrm{kpc} /\left[\tan \left(\phi_{V}^{\max }-\phi_{b}\right)\right] \approx 150 \mathrm{kpc}$. This is evident in the quadrupole moment plots of Figure 3 .

This leads us to consider bounds on the range of acceptable beaming angles $\phi_{b}$. The effective "core radius" in the $\mathrm{HBM}$, is $R_{c} \simeq R_{o} / \phi_{b} \simeq 24\left(\phi_{b} / 20^{\circ}\right)^{-1} \mathrm{kpc}$. This value is confirmed by our numerical results if we define $R_{c}$ as the sampling depth at which $\left\langle V / V_{\max }\right\rangle=0.46$ close to the value found by PVO (Fenimore et al. 1993). Thus by equation 
(11),

$$
R_{c} \simeq 40 V_{3}\left(\frac{\phi_{b}}{\phi_{V}^{\max }}\right)^{-1} \mathrm{kpc},
$$

where $V_{3} \equiv V_{r} / 10^{3} \mathrm{~km} \mathrm{~s}^{-1}$. Since BATSE bounds on $\left\langle\sin ^{2} b\right\rangle$ imply $\left(\phi_{b} / \phi_{V}^{\max }\right)>1$, the HBM cannot have a core radius larger than $\sim 40 V_{3} \mathrm{kpc}$. The minimum core radius that is likely to fit the data is $R_{c} \sim 16 \mathrm{kpc}$ (Paczyński 1991), thus the range of allowed $\phi_{b}$ is roughly

$$
12^{\circ} V_{3}^{-1}<\phi_{b} \leq 30^{\circ}
$$

This agrees with Figure 3, where $\phi_{b}=20^{\circ}$ give the best fit to BATSE data.

Although Fig. 3 is for $V_{r}=1000 \mathrm{~km} \mathrm{~s}^{-1}$, note that better fits to the BATSE data can be obtained when $V_{r}>1000 \mathrm{~km} \mathrm{~s}^{-1}$. This reduces the effect of galactic rotation on the burster distribution, causing $\left\langle\sin ^{2} b\right\rangle$ to converge toward $1 / 3$ at the faint end, and it also widens the acceptable range of $\phi_{b}$ (eq. [3]). Some soft gamma repeaters have recoils as large as $V_{r} \sim 2000 \mathrm{~km} \mathrm{~s}^{-1}$ (Appendix A).

\section{Model Comparisons with the 3B Catalog and PVO}

We now make detailed comparisons of the HBM with the 3rd BATSE catalog (Meegan et al. 1996), which we will refer to simply as "3B". Because the largest deviations of angular moments from isotropy in the HBM occur within bright subsets of bursts (small $D$ in Figure 3), we will explicitly consider the cumulative angular statistics as a function of observed peak photon fluxes, $P$. This is the basic approach that we previously applied to the first and second BATSE catalogs (LDT; LD96a). A preliminary discussion of 3B comparisons is given by LD96b; see also Bulik \& Lamb (1996) for a complimentary statistical analysis. 


\subsection{The $\log \mathcal{N}-\log P$ Distribution}

There are several issues that must be faced when doing statistical analyses of the 3B catalog. There are data gaps in in many bursts, and for many bursts the associated trigger efficiency (the probability that BATSE will detect a burst, as a function of peak flux) is less than one. Under these circumstances, we use the following criteria for selecting bursts from BATSE and PVO: (1) Overwriters in 3B are not included. (2) Only bursts from 3B with $P_{256} \geq 1$ photons $\mathrm{cm}^{-2} \mathrm{~s}^{-1}$, whose trigger efficiency presumably is $\geq 1$, are used. Similarly, bursts from PVO with $P_{250} \geq 25$ photons $\mathrm{cm}^{-2} \mathrm{~s}^{-1}$ are used. (3) Only bursts for which $\left(C_{\max } / C_{\min }\right)_{256} \geq 1$ or $\left(C_{\max } / C_{\min }\right)_{1024} \geq 1$, or both, are used. This applies to both $3 \mathrm{~B}$ and PVO.

This counting criterion reduces the number of BATSE bursts used in our study of the $\log \mathcal{N}-\log P$ distribution to 241 out of the total number 1122 in 3B. This, however, also allows us to construct a relatively homogeneous merged catalog with the PVO data, since the PVO bursts are selected by very similar criteria (Fenimore et al. 1993). Thus we add 181 PVO bursts to our analysis of the $\log \mathcal{N}-\log P$ distribution, making a total of 422. More importantly, by adding PVO we have increased the range of peak fluxes which we can fit by a factor of about 20 .

We have also done analyses by selecting bursts according to criteria (1) and (2), and ignoring criterion (3). This is somewhat justified since the BATSE bursts for which $P_{256}$ and $C_{\max } / C_{\min }$ are available all have $\left(C_{\max } / C_{\min }\right)_{256} \geq 1$ or $\left(C_{\max } / C_{\min }\right)_{1024} \geq 1$ when $P_{256} \geq 1$ photons $\mathrm{cm}^{-2} \mathrm{~s}^{-1}$. This gives us a larger sample, with 437 bursts from BATSE

10 We will use many more 3B bursts in our study of the GRB angular distribution, as described below. Note that a significant fraction of $3 \mathrm{~B}$ bursts do not have $C_{\max } / C_{\min }$ available, so we had to drop these bursts from the start. 
and 198 from PVO, thus making a total of 635. The fitting parameters we obtained from this sample, especially the peak luminosity, are very similar to the values we obtained from fitting the 422 sample, however. Thus we will only present the results from the sample of 422.

The combined PVO and BATSE intensity distribution is shown in Figure 1 . The HBM result for $\phi_{b}=20^{\circ}$ and $V_{r}=1000 \mathrm{~km} \mathrm{~s}^{-1}$ is also shown. We have used two free parameters in fitting these data to the HBM: the absolute number normalization (i.e., the number of GRBs per year) and the intrinsic peak photon emission rate $\dot{N}$. We find a best-fit "standard candle" value of

$$
\dot{N} \simeq 9.45 \times 10^{46}\left(\frac{1-\cos \phi_{b}}{0.06}\right) \text { photons } \mathrm{s}^{-1}
$$

This fit has a minimum $\chi^{2} \simeq 6.9$ with 12 degrees of freedom. The $1-\sigma$ confidence region of $\dot{N}$, estimated as the range over which $\chi^{2}$ increases by 2.3 , is $8.81-11.05 \times 10^{46}$ photons $\mathrm{s}^{-1}$. The corresponding peak luminosity is

$$
\dot{E} \approx 1.5 \times 10^{40}\left(\frac{1-\cos \phi_{b}}{0.06}\right) \operatorname{ergs~s}^{-1}
$$

if the average photon energy is $\sim 100 \mathrm{keV}$. The corresponding BATSE sampling depth is $D_{\text {bat }} \approx 182 \mathrm{kpc}$ for a limiting flux of $\sim 0.3$ photons $\mathrm{cm}^{-2} \mathrm{~s}^{-1}$.

We have also fit the model to 3B alone, ignoring PVO constraints. This is shown in Figure 5. The best-fit peak luminosity turns out to be

$$
\dot{N}=6.9 \times 10^{46}\left(\frac{1-\cos \phi_{b}}{0.06}\right) \text { photons } \mathrm{s}^{-1}, \quad \text { BATSE alone }
$$

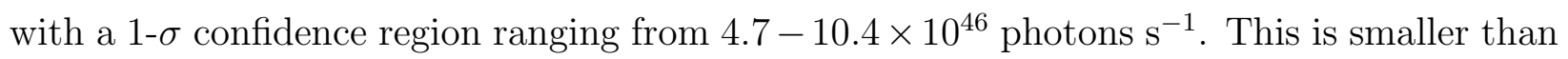
the number we got using the combined PVO and BATSE data, although the confidence regions do overlap. 


\subsection{The Angular Distributions}

In order to compare the HBM with the observed burst angular distributions, we have filtered our model results through BATSE's sky exposure table. All bursts from 3B with tabulated values of $P_{256}$ are used in this comparison except overwriters, giving us 814 bursts in total.

In Figure 6, we plot measures of angular (an)isotropy in galactic-based coordinates for bursts from the $3 \mathrm{~B}$ catalog in which the peak photon flux $\left(P_{256}\right)$ is greater than or equal to a given value, shown on the horizontal axis. Here $\phi_{b}=20^{\circ}$, and we have used the best-fit photon luminosity $\dot{N}$ of equation 4 .

The HBM curves (solid lines) make the distinctive prediction that $\left\langle\cos ^{2} \Theta\right\rangle>1 / 3$, as explained in $\$ 3$. Dashed lines show model predictions if gamma-ray bursts are isotropic on the sky at all intensity levels, as in the cosmological burster hypothesis. Note that both model curves (HBM and isotropy) are corrected for the incomplete BATSE sky coverage. The numerical (Monte Carlo) uncertainties on the HBM curves are very small on the scale of this graph: comparable to the width of the thick solid lines.

Filled dots with error bars in Figure 6 are BATSE cumulative data points with $1-\sigma$ statistical uncertainties evaluated according to

$$
\sigma(\langle X\rangle)=N^{-1 / 2}\left[\left\langle X^{2}\right\rangle-\langle X\rangle^{2}\right]^{1 / 2}
$$

(e.g., Briggs 1993) where $X \equiv \cos \Theta, \sin ^{2} b$ or $\cos ^{2} \Theta$, and the averages $\langle X\rangle$ and $\left\langle X^{2}\right\rangle$ are taken in the HBM. These error bars, appropriate for comparing observations with the HBM (solid lines), are slightly larger than the error bars that would be appropriate for comparing with the isotropic model (dashed lines).

11 Briggs et al. (1996) have studied the angular distributions of 1005 GRBs, putting 
Figure 6 shows that the 3B catalog is statistically consistent with both isotropy and the HBM at the $2-\sigma$ level or better, for all angular statistics and at all peak photon fluxes. Nevertheless, isotropy fits the observations better than the HBM in every graph, unlike in our earlier studies based on 1B and 2B (LDT; LD96a). At the bright end, the HBM offers several clear predictions which may be tested as BATSE accumulates more data.

\subsection{Short \& Long Burst Subclasses in the HBM}

When the numbers of BATSE bursts are plotted as a function of burst duration (i.e., a histogram), they show a statistically significant two-peaked distribution (Kouvelioutou et al. 1993; Koshut et al. 1996; Meegan et al. 1996). This suggests that GRBs can be physically grouped into two classes. In this paper we have analyzed the distribution of short and long bursts together, using the photon peak flux on a $0.256 \mathrm{~s}$ time scale, $P_{256}$, as a brightness measure in order to make a homogeneous merged set of data from BATSE and PVO. However, in two earlier papers (LDT and LD96a), we studied the BATSE duration subclasses independentally, and compared them to HBM predictions. This was useful for exploring the possibility that the HBM, or indeed any galactic halo model, might

constraints on galactic models, including the HBM. They found a deviation of $3.1 \sigma$ between the HBM and BATSE values of $\left\langle\cos ^{2} \Theta\right\rangle$ for the brightest $1 / 8$ of their sample, which is larger than we find here. Our analysis differs from Briggs et al. in two ways. First, a new intrinsic peak luminosity was found from fitting 3B/PVO (§4.1), which is slightly larger than the luminosity that DLT found from fitting 1B/PVO, as used by Briggs et al. Second, our statistical uncertainties come from equation (7) where the averages $\left\langle\cos ^{2} \Theta\right\rangle$ and $\left\langle\cos ^{4} \Theta\right\rangle$ are evaluted in the HBM rather than in isotropic models. Isotropic models underestimate the relevant uncertainties by more than $10 \%$. 
apply to one subclass only. In these studies we used $P_{1024}$ for the long bursts $\left(T_{90}>2 \mathrm{~s}\right)$ and $P_{64}$ for the short bursts $\left(T_{90}<2 \mathrm{~s}\right)$. The peak luminosity obtained for the $2 \mathrm{~B}$ long

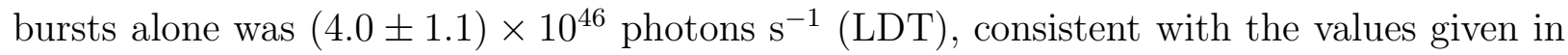
eqn. (6). Our plots of GRB angular statistics as a function of burst brightness for the two subclasses (analogous to Figure 6) showed that the BATSE data was fit by the HBM and isotropic (cosmological) models almost equally well. No statistically significant deviations from isotropy or from the HBM were found in either subclass at any peak flux level.

\section{Burster Energy Budgets and Repetition Rates}

If halo GRB sources are observed as soft gamma repeaters (SGRs) when they are young, then a rough lower bound on the galactic formation rate of bursters is $\Gamma>10^{-4}$ $\mathrm{yr}^{-1}$. This is based on the fact that 3 SGRs have been detected in likely association with supernova remnants of age $\sim 1 \times 10^{4}$ years. Note that SGR activity is episodic (e.g., SGR 0526-66 was observed to burst only during the years 1979-1983; Norris et al. 1991), and only a small fraction of Type II supernova remnants have confirmed radiopulsar (non-SGR [2) counterparts (Frail 1997; Frail \& Moffett 1993), thus there might exist many heretofore undetected SGRs of ages $\lesssim 10^{4}$ yrs in the galaxy. Parameterizing $\Gamma_{3} \equiv\left(\Gamma / 10^{-3}\right.$ $\mathrm{yr}^{-1}$ ), we find:

$$
0.1<\Gamma_{3}<10
$$

${ }^{12}$ The $P_{\text {rot }}=8.0$ s rotation period and other properties of SGR 0526-66 clearly indicate that SGRs are, as a class, distinct from rotation-powered pulsars. Only one SGR, 1806-20, is embedded in a radio plerion, and this has a steeper spectrum than a pulsar plerion (Kulkarni et al. 1994). This plerion might be magnetically-powered, as explained in Appendix A. 
where the upper bound comes from the fact that the rate of burster (SGR) formation cannot exceed the total galactic supernova rate.

If the BATSE sampling depth is $D=100 D_{100} \mathrm{kpc}$ and if all bursters acquire recoils of magnitude $\sim 1000 V_{3} \mathrm{~km} \mathrm{~s}^{-1}$ (cf. Appendix A), then the time for a burster to drift out to the BATSE sampling depth is $t_{D}=10^{8} D_{100} V_{3}^{-1}$ yrs. The total number of bursters in the volume sampled by BATSE is then

$$
\mathcal{N}_{D}=\Gamma t_{D}=10^{5} D_{100} V_{3}^{-1} \Gamma_{3}
$$

It is worthwhile emphasizing the meaning of this equation. Given the observed SGR formation rate [eq. (8)] and their observed recoils (Appendix A) we can conclude on a purely empirical basis that there exists a galactic halo of $>10^{4}$ aged SGRs within the distance 100 kpc (DT92). The number of old SGRs in the halo might be orders of magnitude higher if $\Gamma_{3}>0.1$, or if a significant fraction of SGRs remain bound to the galaxy (eq. [26] below). SGRs are known to emit multiple soft gamma-ray bursts of energy $\sim 10^{41}$ ergs when they are young; if they continue to emit bursts with similar energies but harder spectra as they age, then they constitute halo GRB sources (DT92).

The (extrapolated) full-sky rate of bursts that are bright enough to be detected by BATSE is $\sim 800 \mathrm{yr}^{-1}$ (Meegan et al. 1992). If emitted by aged SGRs, then the mean repetition time for bursts from a given star is 3

$$
\tau_{G R B} \simeq 110 \Gamma_{3} V_{3}^{-1} D_{100 \mathrm{yrs}}
$$

This implies that evidence for repetition might be difficult to find in presently available data.

${ }^{13}$ In the HBM, the beaming of bursts reduces the number of observable bursters by only a mild factor $f \approx 1-(2 / 3) R_{o} /\left(\phi_{b} D\right) \approx 1-0.16 D_{100}^{-1}\left(\phi_{b} / 20^{\circ}\right)^{-1} \approx 0.84$, where $R_{o} \sim 8.5 \mathrm{kpc}$ is a galactic disk dimension. This (unimportant) factor is included eq. (10). 
The intrinsic luminosity of a GRB near the BATSE detection threshold of $F_{\text {BATSE }} \simeq 1 \times 10^{-7} \mathrm{erg} \mathrm{cm}^{-2} \mathrm{~s}^{-1}$ (Meegan et al. 1992) is

$$
\dot{E}_{\mathrm{GRB}} \simeq 1 \times 10^{41} D_{100}^{2}\left(\frac{\Delta \Omega}{4 \pi}\right) \mathrm{erg},
$$

where $\Delta \Omega$ is the solid angle of the burst emissions. This agrees with the luminosity quoted in equation (5), for a best-fit BATSE sampling depth $D_{100}=1.82$ and $\phi_{b}=20^{\circ}$. The effective duration of BATSE GRBs is $\sim 10 \mathrm{~s}$, as found from the mean ratio of fluence to peak flux (Lee \& Petrosian 1996). Thus the burst energy is

$$
E_{\mathrm{GRB}} \simeq 1 \times 10^{42} D_{100}^{2}\left(\frac{\Delta \Omega}{4 \pi}\right) \mathrm{erg} .
$$

In its observable (by BATSE) lifetime of $t_{D}=10^{8} D_{100} V_{3}^{-1}$ yrs, each star emits a number of bursts $\mathcal{N}_{B}=t_{D} / \tau_{G R B}=10^{6} \Gamma_{3}^{-1}$. The total energy required from each burster is then $E_{\mathrm{tot}}=\mathcal{N}_{B} E_{\mathrm{GRB}}$, or

$$
E_{\text {tot }} \simeq 3 \times 10^{46} D_{100}^{2} \Gamma_{3}^{-1}\left(\frac{1-\cos \phi_{b}}{0.06}\right) \mathrm{erg} .
$$

This is the basic energy requirement for burst sources in the HBM. Note that in the small-angle limit四

$$
\left(\frac{1-\cos \phi_{b}}{0.06}\right) \approx\left(\frac{\phi_{b}}{20^{\circ}}\right)^{2}, \quad \phi_{b} \ll 1 .
$$

\footnotetext{
${ }^{14}$ In the remainder of $\S 5$ and in $\S 6.3$ we will quote many results with a scaling factor $\left(\phi_{b} / 20^{\circ}\right)^{2}$. These equations are given implicitly in the $\phi_{b} \ll 1$ limit. However, all these results can be generalized to any $\phi_{b}$ by using eq. (14). In particular, a magnetar model with isotropic $\left(\phi_{b}=\pi / 2\right)$ bursters could agree with BATSE and PVO data if the initial onset of bursting were delayed (LD92; Bulik \& Lamb 1996; Appendix B, eq. [B7]). Energy budget constraints for this scenario are given by dropping the $\left(\phi_{b} / 20^{\circ}\right)^{2}$ factor in eq. (17) and multiplying by a numerical factor $(0.06)^{-1}=16.6$. These same isotropic results would apply if bursts were beamed, but in random directions; for example, if the alignment scenarios of
} 
Rotational energy, the power source for ordinary pulsars, is probably not capable of providing this much energy for GRBs (LD92; Hartmann \& Narayan 1996). Furthermore, there is evidence that SGRs are slow rotators $\left(P_{\text {rot }}=8.0 \mathrm{~s}\right.$ for SGR 0526-66). We now discuss more promising energy sources.

\subsection{Magnetic Energy}

A neutron star with interior flux density $B=10^{16} B_{16} \mathrm{G}$, idealized as uniform throughout the star, has magnetic energy

$$
E_{B}=B^{2} R^{3} / 6=2 \times 10^{49} B_{16}^{2} \text { erg. }
$$

This is a plausible estimate for the magnetic energy of a young neutron star with an internal toroidal field generated by a saturating $\alpha-\Omega$ dynamo (DT92; TD93). It exceeds by a factor $\sim 10^{3} \Gamma_{3}$ the GRB energy requirement of eq. (13). The transition to superconductivity occurs at $B<B_{c} \sim 3 \times 10^{15} \mathrm{G}$. When $B<B_{c}$, magnetic flux is concentrated by supercurrents into discrete flux tubes, and the energy is (PRR):

$$
E_{B}=B B_{c} R^{3} / 6=5 \times 10^{47} B_{15} \mathrm{erg} .
$$

As a magnetar field evolves and decays it will enter the regime of eq. (16), even if it is in the eq. (15) regime initially.

Equations (13) and (16) together imply that magnetic energy can suffice to produce all GRBs in the HBM if the burster internal magnetic field satisfies

$$
B>6 \times 10^{13} D_{100}^{2} \Gamma_{3}^{-1}\left(\frac{\phi_{b}}{20^{\circ}}\right)^{2} \mathrm{G} .
$$

$\S 2.2$ did not operate. Although the energy per burst would then be reduced by $(\Delta \Omega / 4 \pi)$,

one would need $(\Delta \Omega / 4 \pi)^{-1}$ more bursts to produce the same rate of observable events; these factors cancell in the energy budget (Hartmann \& Narayan 1996). 
This criterion is met, and in fact exceeded by a factor $\sim\left(10-10^{3}\right) \Gamma_{3}$, in magnetar formation scenarios (DT96) and in the magnetar model of SGRs (TD95, TD96).

Regardless of the energy source for bursts, if the energy release occurs inside a neutron star and is communicated outward by material and Alfvèn waves (e.g., Blaes et al. 1989), then very strong magnetic fields, $B>B_{Q}$ are probably necessary. Weaker fields would allow vibrational crumbling of a halo neutron star's crust and thereby produce an X-ray excess (Appendix B).

\subsection{Accretion Energy}

The sudden accretion of a planetesimal (asteroid or comet) with mass

$$
M_{p}=5 \times 10^{21} D_{100}^{2} \epsilon_{\gamma}^{-1}\left(\frac{\Delta \Omega}{4 \pi}\right) \mathrm{gm}
$$

onto a neutron star would release the energy of a halo GRB, where $\epsilon_{\gamma}$ is the efficiency of gamma ray production. This is a scaled-up version of the GRB theory of Harwit \& Salpeter (1973) and Colgate \& Petchek (1981).

To power all $10^{6} \Gamma_{3}^{-1}$ bursts in a burster's observable lifetime, the total mass of accreted planetesimals is

$$
M_{\mathrm{tot}}=5 \times 10^{27} \Gamma_{3}^{-1} D_{100}^{2} \epsilon_{\gamma}^{-1}\left(\frac{\Delta \Omega}{4 \pi}\right) \mathrm{gm},
$$

similar to the mass of the Earth. These planetesimals presumably condense from a gaseous disk. Such a disk $\left(M_{\text {disk }}>M_{\text {tot }}\right)$ could be captured by a HVNS when it overtakes clumpy supernova ejecta (Woosley \& Herant 1996) or experiences a recoil directed toward a stellar companion (Colgate \& Leonard 1994). Alternatively, a disk could form when a rapidly-rotating proto-neutron star sheds excess angular momentum (e.g., Thompson \& Duncan 1994). In this case, the intial radius of the retained disk could not exceed 
$R_{i, \text { disk }} \sim\left(G M_{\star} / V_{r}^{2}\right) \sim 2 \times 10^{5} V_{3}^{-2} \mathrm{~km} ;$ but it would subsequently experience viscous spreading.

It is not obvious how the beaming and alignment requirements of the HBM are met in such an accretion-powered scenario, although the physics is complex enough that a mechanism might be identified. Alternatively, GRBs could be unbeamed, or beamed in random directions, but with a delayed onset of bursting (LD92). Colgate \& Leonard (1994) estimated that a time interval $\sim 10^{7}$ years, as required to fit BATSE data, occurs before planetesimals condense in the disk. In the pulsar-planet formation scenario of Lin, Woosley \& Bodenheimer (1991), some objects as large as $\sim 10^{21} \mathrm{gm}$ formed much earlier, after $\sim 10^{3}$ yrs, but $\gtrsim 10^{6}$ yrs were needed to form planets. Planets might be necessary to deflect a significant number of objects toward the neutron star (e.g., van Buren 1981; Tremaine \& Żytkow 1986), so a delayed turn-on as Colgate \& Leonard propose seems feasible.

Although the gross energy requirements of halo GRBs can easily be met in an accretion-powered model, these models have two potential problems: (1) producing hard non-thermal gamma ray spectra without over-producing thermal X-rays; and (2) producing a large enough rate of abrupt planetesimal captures (eq. [10]).

Some form of magnetically-mediated gamma-ray production is usually invoked to solve the first problem (e.g., Colgate 1992). That is, the accreting matter distorts the magnetic field, which - via nonlinear Alfvén wave damping, tearing instabilities, or some other mechanism-accelerates charged particles to produce gamma rays. Note, however, that the total exterior magnetic energy of a neutron star with $B_{\text {dipole }}=10^{12} B_{12} \mathrm{G}$ is less than the energy of a single GRB in the galactic halo by

$$
\frac{E_{B}}{E_{G R B}}=0.2 B_{12}^{2} D_{100}^{-2}\left(\frac{\Delta \Omega}{4 \pi}\right)^{-1} .
$$

It is difficult to understand how such a relatively large accretion energy (eq. [12]) could be channeled through such a weak field without enormous inefficiency, resulting in the 
over-production of thermal X-rays. This problem is discussed in more detail in $§ 7.3 .1$ of TD95.10

A strong magnetic field is also generally invoked to increase the capture cross-section of a neutron star, thereby enhancing the putative GRB rate (Colgate \& Petchek 1981; van Buren 1981). A crude estimate of the radius $R_{c}$ at which magnetic capture will occur in a single orbital pass is given by the condition that the kinetic energy of mass $M_{p}$ at $R_{c}$ be comparable to the magnetic dipole field energy external to $R_{c}$. This yields

$$
\frac{R_{c}}{R_{\star}}=\left(\frac{R_{\star}^{4} B_{\text {dipole }}^{2}}{6 G M_{\star} M_{p}}\right)^{1 / 2}=1.7 B_{12} D_{100}^{-1} \epsilon_{\gamma}^{1 / 2}\left(\frac{\phi_{b}}{20^{\circ}}\right)^{-1}
$$

for $R_{\star}=10 \mathrm{~km}$ and $M_{\star}=1.4 M_{\odot}$. Thus the single-orbit capture cross-section is barely enhanced over the geometrical cross-section of the star for pulsar-strength fields and halo locations. Multi-orbit magnetic captures also occur (van Buren 1981); but as shown by Woosley \& Herant (1996), very oblique captures produce extended soft X-ray events.

For local galactic disk bursters $\left(D_{100} \sim 10^{-3}\right)$, the context in which planetesimal accretion was originally proposed, neither of these problems (as roughly illustrated by the small numbers in eq. [20] and [21]) are serious. However, for halo GRBs, dramatic beaming $(\Delta \Omega \ll 1)$ or magnetic fields much stronger than $10^{12} \mathrm{G}$, or both, seem theoretically desirable.

Thus we suggest that planetesimal accretion onto magnetars may power halo GRBs. Some subset of the rapidly-rotating proto-neutron stars which become magnetars (according to the conjecture described in DT96) may shed excess angular momentum into low-mass disks (eq. [19), or capture disk material as they move through clumpy supernova ejecta (Woosley \& Herant 1996). The exterior magnetic energy of these stars is greater than the

\footnotetext{
${ }^{15}$ TD95 focus on accretion-powered models of the 1979 March 5th event's hard initial spike, but the spectral requirements are similar (FKL).
} 
energy of a halo GRB by $\sim 2 \times 10^{5} B_{15}^{2} D_{100}^{-2}(\Delta \Omega / 4 \pi)^{-1}$, potentially catalyzing substantial non-thermal emissions. The single-orbit capture cross-section for planetesimals is enhanced over the geometrical cross section by a similar factor.

The HBM could apply in a magnetar-accretion model because the mechanisms for stellar recoils and for aligned beaming described in $\S 2$ would hold. Alternatively, a time delay for planetesimal formation (Colgate \& Leonard 1994) may operate (LD92). This physical model for halo GRBs is viable even if all magnetically-powered activity terminates after the SGR phase, as long as a magnetar retains a substantial fraction of its initial dipole field, anchored in the stably-stratified core, for a time $\gtrsim 10^{8} V_{3}^{-1} D_{100}$ years.

\section{Model Extensions and Refinements}

\subsection{Intensity Variations Across the Beam}

So far we have assumed that the gamma ray intensity is uniform within a beaming cone of half opening-angle $\phi_{b}$. In this section we will study the implications of a gamma-ray intensity which varies as a function of the angle from the beam axis.

We will use the relativistic beam model of Mao \& Yi (1994). In this model, gamma-ray emitting material is expanding with Lorentz factor $\gamma \equiv\left(1-v^{2} / c^{2}\right)^{-1 / 2}$ into a cone with half opening angle $\Delta \theta$. Emissions are assumed to be isotropic in the rest-frame of the emitting material, which means that they are beamed to within an opening angle $\sim \gamma^{-1}$ about the material velocity vector in the observer's frame. Integrating over the material beam and over photon emission angles, Mao \& Yi (1994) derived the net observable photon intensity as a function of angle $\theta$ away from the beam axis:

$$
I(\theta)=\Lambda \int_{0}^{2 \pi} d \phi^{\prime} \int_{0}^{\Delta \theta} \sin \theta^{\prime} d \theta^{\prime} \chi[\gamma(1-[v \chi / c])]^{-(2+\delta)}
$$


In this equation, $\chi \equiv \cos \theta^{\prime} \cos \theta-\sin \theta^{\prime} \sin \phi^{\prime} \sin \theta ; \delta$ is the photon spectral index (we will take $\delta=2$ ); and $\Lambda$ is a normalization factor, which we will define such that $I(0)=1$ for all values of $\Delta \theta$ and $\gamma$.

This idealized physical model provides a useful two-parmeter family of beams with centrally-peaked brightness. Yi and Mao (1994) used this model to show that relativistic beaming effects in galactic halo GRB models can produce anti-correlations between the brightness and duration of observed bursts, mimicking cosmological time dilation.". Such correlations were found by Norris et al. (1993) in the BATSE data, but not by Mitrofanov et al. (1996).

In Figure 7 we plot $I(\theta)$, as given by equation (22). We have kept $\Delta \theta=20^{\circ}$ in this plot, which ensures that the beam half-width at half-maximum stays close to $20^{\circ}$. (This is likely to fit BATSE and PVO data best; see eq. [3].) Figure 7 shows that, for small $\gamma$, bursters become potentially detectable at large $\theta\left(>20^{\circ}\right)$, although only at low intensity, i.e., only within a diminished sampling depth by BATSE. The $\phi_{b}=20^{\circ}$ uniform beam case, considered in $\S 3$ and $\S 4$ (solid line in Figure 7 ), is equivalent to the limit $\gamma \rightarrow \infty$ on this plot.

What values of $\gamma$ are plausible for a halo GRB model? A pure photon/pair fireball (presumably channeled by the magnetic field into a restricted solid angle) with intrinsic luminosity appropriate for a galactic halo GRB would have $\gamma \sim 10$ or slightly less (Piran \& Shemi 1993). This is comparable to the value used by Yi and Mao (1994) in their halo "time dilation" study. The Lorentz factor could be moderately reduced, say to $\gamma \sim 3$, by a

\footnotetext{
${ }^{16}$ When bursters are unbound from the Galaxy, as in the HBM, more distant sources are systematically older. Any trend of spectral softening or increasing burst duration with age could then mimic cosmological time dilation.
} 
plausible contamination of baryons. [T]

To study the implications of these $I(\theta)$ models, we assume as before that the axi of beamed GRB emissions are aligned with peculiar recoil velocities $\mathbf{V}_{\mathbf{r}}$ in a galactic population of bursting stars, and we do new Monte Carlo calculations of the observable GRB distributions. Specifically, for all the $I(\theta)$ functions shown in Figure 7, we repeated the $\log \mathcal{N}-\log P$ model fitting against PVO and 3B data (as shown in Figure 4 for the uniform beam case), finding the best-fit peak photon luminosity, now defined at the beam center $(\theta=0)$. We then used this best-fit value to calculate the cumulative angular statistics as a function of peak photon flux, similar to Figure 6. We show these results in Figure 8. For clarity, we have plotted the difference of the angular statistics $\langle\cos \Theta\rangle,\left\langle\sin ^{2} b\right\rangle$ and $\left\langle\cos ^{2} \Theta\right\rangle$ from the values predicted in the uniform beam case (i.e., the $\phi_{b}=20^{\circ} \mathrm{HBM}$ values, as plotted in Figure 6), for various intinsic beaming patterns $I(\theta)$.

Figure 8 shows that, as long as the half-width at half-maximum of the gamma-ray beam is held constant, observable results for various beam intensity patterns $I(\theta)$ deviate little from the uniform beam case. (Note that the vertical axis scale in Figure 8 is expanded by a factor $\sim 10$ relative to that of Figure 6.) This is our main conclusion. In fact, all of the $I(\theta)$ beaming patterns considered in Figure 7 and Figure 8 fit the present 3B data with better than 3- $\sigma$ confidence at all observed peak photon flux levels, as can be seen by comparing Figure 8 with the BATSE data of Figure 6. Thus a uniform beam is not required in the HBM, as long as the variation within the beam is not too large.

${ }^{17}$ Of course, fireballs produce (blueshifted) thermal-spectrum photons, which is not what GRBs look like; however this gives at least a first estimate of what the hydrodynamicallydriven Lorentz factor would be. Electrodynamic acceleration (e.g., driven by magnetic reconnection or Alfvén waves) might produce larger $\gamma$, and/or different beam patterns. 


\subsection{Burst Luminosity Functions}

We now relax the "standard candle" assumption (point [4] in the second paragraph of §2.1). We will consider GRB luminosity functions of the form

$$
\Phi(L) \propto L^{-\beta} \quad \text { for } \quad L_{\min }<L<L_{\max } .
$$

Constraints on GRB luminosity functions have been previously inferred from BATSE and

PVO data by Ulmer, Wijers \& Fenimore (1995, hereafter UWF), Hakkila et al. (1995), and by other workers cited therein. In these studies, the bursters' spatial density distribution in the galactic halo was assumed to be

$$
\rho(r)=\frac{\rho_{0}}{\left[1+\left(r / R_{c}\right)^{\alpha}\right]} .
$$

The intensity distribution of GRBs $(\log \mathcal{N}-\log P$ curve) in the HBM resembles that of the $\alpha=2$ case of this model, although the angular distribution of observable bursters as a function of $r$ is very different.

These previous studies concluded that the acceptable dynamic range $\left(L_{\max } / L_{\min }\right)$ for GRBs observed by BATSE is quite limited. In particular, various studies agree that $90 \%$ of detected bursts must have peak luminosities within a factor of $\sim 10$, for a wide range of indices $\alpha$ and $\beta$. The basic reason for this is that the turnover of the observed $\log \mathcal{N}-$ $\log P$ curve (Fig. 4) from a slope $d \log \mathcal{N}(>L) / d \log L=-3 / 2$ (the "Euclidean core") to a shallower slope at fainter fluxes happens rather sharply. A GRB luminosity function that spanned much more than one order of magnitude would smooth out this turnover to an extent inconsistent with observations.

In the $\alpha=2$ case (relevant to the HBM) UWF found, furthermore, that the dynamic range of the luminosity function, $L_{\max } / L_{\min }$, can only be as large as $\sim 10$, and still fit observations, if the power-law index lies in the range $\beta=2-3$. (See Fig. 2 in UWF). Other values of $\beta$ would require significantly more restricted dynamic ranges. This result 
can be understood analytically. If we assume $L_{\max } / L_{0}=L_{0} / L_{\min } \equiv \kappa$ where $L_{0}$ is the best standard-candle (mono-luminosity) fit to the data, then simple integrals show that the average luminosity satisfies $\bar{L} / L_{0} \approx 1$ for $2<\kappa<20$ only when $\beta \sim 2-3$. $\bar{L} / L_{0}$ is very different from unity when $\kappa>3$ for all other values of $\beta$. Since luminosity functions with $\bar{L} / L_{0} \sim 1$ give the best fits to observations, the dynamic range, given by $\kappa^{2}=L_{\max } / L_{\min }$, can only be large for $2<\beta<3$. More general analytic constraints on GRB luminosity functions have been derived by Horak et al. (1996).

These above-quoted results were all obtained from fits to the the observed $\log \mathcal{N}-\log P$ curve only. Next we consider constraints on the GRB luminosity function from the observed $\log \mathcal{N}-\log P$ distribution combined with the observed angular distributions. These combined constraints were previously considered by Hakkila et al. (1995) for a galactic halo of bursters with density given by eqn. (24) and no preferred beaming directions. Here we focus on the very different case of the HBM. (Of course, the GRB angular distribution cannot constrain the luminosity function of cosmological GRBs, since the bursters are distributed essentially isotropically around Earth at all intensity levels.)

Intuitively, luminosity functions could either wash out or enhance the angular anisotropies predicted by HBM for bright subsets of GRBs (Fig. 6). If the luminosity function of bursts is a positive (negative) power-law, then at a given observed flux level, a greater (smaller) number of more distant bursters are counted than in the mono-luminosity case. Consequently, the degree of anisotropy is reduced (enhanced) since the HVNS distribution appears more and more isotropic at greater distances from the Earth.

To illustrate this, we consider two cases: $(\beta, \kappa)=(-3,2),(3,4.4)$. Both models give acceptable fits to the $\log \mathcal{N}-\log P$ distribution of the combined PVO and BATSE data, but the allowed dynamic range $L_{\max } / L_{\min }=\kappa^{2}$ is much smaller in the $\beta=-3$ case, for reasons discussed above. Figure 9 shows corresponding angular distribution statistics 
(dashed and dotted curves) compared to the mono-luminosity case (thick solid curve). It is clear that the luminosity function with positive slope $(-\beta=3)$ affords a better fit to the angular position data than the standard-candle model does. For $\beta=3$, on the other hand, the angular statistics deviate from BATSE by $\sim 3-\sigma$ at the faint end. (Plotted error bars are $3-\sigma$.

We conclude that only a limited spread of intinsic luminosities among GRBs observed by BATSE and PVO are admissible in the HBM, as in other galactic halo models. Depending on the exact form of the luminosity function, this could either improve or worsen the model's fit to the BATSE angular distribution. However, in either case the effect is limited because the dynamic range is small. Equivalently, one could say that in a fit to the combined GRB brightness and angular position data, angular information increases (decreases) the statistically-allowed dynamic range of the luminosity function when $\beta<0$ $(\beta>0)$, but this dynamic range is less than $\sim 10$ in any case.

Note that we have considered only one possible generalization of the basic (standard) HBM here: a power-law luminosity function that is uniform among bursters. Other conceivable generalizations, such as luminosity functions that vary with burster age, or luminosities correlated with $\phi_{b}$ or $V_{r}$, would produce different results.

\subsection{Weakly-bound Bursters in the HBM}

Next we consider relaxing the assumption that all bursters are born with $V_{r}=1000$ $\mathrm{km} \mathrm{s}^{-1}$ (point [2] in the second paragraph of $§ 2.1$ ). If some fraction of bursters are born with velocities less than the local galactic escape velocity, which is about $V_{\text {esc }} \sim 600 \mathrm{~km} \mathrm{~s}^{-1}$ at typical birthsites in the disk, these stars will orbit the galaxy. Podsiadlowski, Rees and Ruderman (1995) have emphasized the important point that these weakly-bound bursters 
do not return to the small galactic radii at which they were born because the gravitational potential in the halo is probably markedly non-spherical. Large deviations from spherical symmetry are predicted by almost all models of galactic halo formation (e.g., Dubinski 1992 and references therein). Thus weakly-bound bursters (i.e., bursters born with velocities narrowly below the local galactic escape velocity) will tend to stay in the outer halo, following wide-ranging, non-periodic orbits. After one or more orbits, their magnetic axi orientations are randomized. If these weakly-bound bursters continue to emit (beamed) bursts, their contribution to observed GRBs must be added to the bursts from the escaping ones (i.e., the unbound "standard HBM" sources, studied in $\S 3$ and $\S 4$ ). This could, under some conditions that we will discuss below, produce a net angular distribution of GRBs that is more isotropic than that of the standard HBM, especially at the faint end. However the basic HBM mechanism for producing the "Euclidean core" in the GRB brightness distribution, as observed by PVO, may still apply.

To quantify this discussion, assume that a fraction $f=10^{-1} f_{-1}$ of all bursters are born with velocities below the local galactic escape velocity, and the remainder have $V_{r}=1000 V_{3}$ $\mathrm{km} \mathrm{s}^{-1}$ as before. This is a crude way to estimate the effects of a realistic (Gaussian) velocity distribution. If the total birthrate of GRB sources in the galaxy is $\Gamma=10^{-3} \Gamma_{3}$ $\mathrm{yr}^{-1}$ (eqn. [8]), then the number of bound bursters presently orbiting in the halo is

$$
\mathcal{N}_{\text {bound }}=\Gamma f \tau_{\text {gal }}=1 \times 10^{6} \Gamma_{3} f_{-1}
$$

where $\tau_{\text {gal }} \sim 10^{10} \mathrm{yr}$ is the age of the galaxy (i.e., the period during which bursters have been forming). The relative abundance of bound bursters compared to unbound ones (eqn. [9]) within the BATSE sampling depth of $D=100 D_{100} \mathrm{kpc}$ is then

$$
\frac{\mathcal{N}_{\text {bound }}}{\mathcal{N}_{\text {unbound }}}=10 f_{-1} D_{100}^{-1} V_{3} \text {. }
$$

Thus if $>1 \%$ of bursters remain bound to the galaxy $\left(f>10^{-2}\right)$, bound stars will numerically dominate the halo (DT92). If the bursters continue to emit bursts within 
beaming cones of half angle $\phi_{b}$, but with orientations randomized by their orbits, for a bursting lifetime $\tau_{\text {life }}=10^{10} \tau_{10}$ years, then the fraction of all bound bursters that are observable at Earth is

$$
\left(1-\cos \phi_{b}\right) \frac{\tau_{\text {life }}}{\tau_{\text {gal }}}=0.06 \tau_{10}\left(\frac{\phi_{b}}{20^{\circ}}\right)^{2} .
$$

Multiplying equation (26) and equation (27) yields the relative number of observable bound bursters compared to unbound ones:

$$
\Psi_{b}=0.6 f_{-1} \tau_{10} D_{100}^{-1} V_{3}\left(\frac{\phi_{b}}{20^{\circ}}\right)^{2}
$$

It is interesting that this can be of order unity.

A fraction $\Psi_{b} /\left(1+\Psi_{b}\right)$ of all observed GRBs will come from bound (orbiting) bursters. How anisotropic will be the sky distribution of these "bound bursts"? This depends upon several uncertain factors, especially the burster recoil velocity distribution and the form of the galactic halo potential. Here we limit ourselves to a few general comments, based upon the results of PRR.

If many bursters are born with $V_{r} \lesssim 500 \mathrm{~km} \mathrm{~s}^{-1}$, significantly below the escape velocity from the center of the galaxy, then the bound burst distribution will be very anisotropic (cf. Fig. 2a and 2b of PRR). If, however, most bound bursters aquire velocities only narrowly below the local escape velocity, then the bound burster distribution could be nearly isotropic, as shown in Fig. 5b of PRR. This figure shows the burster distribution for kick velocities uniformly distributed on the range $700 \leq V_{r} \leq 900 \mathrm{~km} \mathrm{~s}^{-1}$, and a realistic, nonspherical galactic potential.

In the magnetar model of GRBs, burster recoils are imparted by a complicated series of magnetically-induced neutrino impulses (§2.2), with durations comparable to the coherence time of magnetic structures near and below the neutrinosphere (DT92; TD93). Because these impulses are additive, the central limit theorem implies that a Gaussian with mean 
$\bar{V}$ and variance $\sigma_{V}$ is probably a good first approximation to the $V_{r}$ distribution among bursters. The precise values of $\bar{V}$ and $\sigma_{V}$ are uncertain, although SGR data suggest $\bar{V} \gtrsim 1000 \mathrm{~km} \mathrm{~s}^{-1}$ (Appendix A). Thus the $V_{r}$ distribution for bound bursters is probably the low $-V_{r}$ tail of a Gaussian. If $\sigma_{V} \lesssim 200-300 \mathrm{~km} \mathrm{~s}^{-1}$, then the velocity distribution of bound bursters is sharply peaked just below the escape velocity, and conditions for a nearly isotropic sky distribution of bound bursts could be met. The distribution of bound bursters might still show mild "hot spots" in some presently-unknown directions associated with distortions in the outer galactic halo potential (PRR), but these anisotropies might not be apparent from the statistical tests used in this paper. [More general statistics (e.g., Briggs et al. 1996; Tegmark et al. 1996) would be useful for constraining this effect.]

Assuming that $\sigma_{V} \lesssim 200-300 \mathrm{~km} \mathrm{~s}^{-1}$ and that other conditions for a nearly-isotropic bound burst distribution are met, then the total model galactocentric quadrupole moment would be

$$
\left\langle\cos ^{2} \Theta\right\rangle-1 / 3=\left(\Psi_{b}+1\right)^{-1}\left[\left\langle\cos ^{2} \Theta\right\rangle_{\mathrm{HBM}}-(1 / 3)\left(1-\Psi_{b}\right)\right]
$$

where $\left\langle\cos ^{2} \Theta\right\rangle_{\text {HBM }}$ is the moment in the "standard HBM", as shown in Figure (6), and we have assumed that the bound burster distribution has $\left\langle\cos ^{2} \Theta\right\rangle=1 / 3$. Thus for $\Psi_{b}=1$, the deviation from isotropy would be reduced by $(1 / 2)$ compared to the standard HBM. Similar results might apply for $\langle\cos \Theta\rangle$ and $\left\langle\sin ^{2} b\right\rangle$; however it is also possible that $\sigma_{V} \gtrsim 300$ $\mathrm{km} \mathrm{s}^{-1}$ and that the addition of bound stars makes the model fits to BATSE observations worse. We will study this issue in a future paper.

Note that the mean time between bursts from a given star is longer by a factor $\left(1+\Psi_{b}\right)$ when a population of bound bursters is present:

$$
\tau_{G R B}=110\left(1+\Psi_{b}\right) \Gamma_{3} V_{3}^{-1} D_{100} \mathrm{yrs}
$$

(cf. eq. [10]). Despite this fact, the energy required for each burster is significantly greater, 
because of the long bursting lifetime:

$$
E_{\text {tot }}=5 \times 10^{48} \tau_{10} D_{100} \Gamma_{3}^{-1} V_{3}\left(1+\Psi_{b}\right)^{-1}\left(\frac{\phi_{b}^{2}}{20^{\circ}}\right)^{2} \mathrm{erg} .
$$

This still might be supplied by magnetic energy (cf. eq. 15]). Alternatively, if the interior flux density satisfies $B<3 \times 10^{15} \mathrm{G}$ initially, then from eq. (16) and eq. (31) we infer that magnetically-powered bursting would have to terminate after an active lifetime no longer than

$$
\tau_{\text {life }}=10^{9} B_{15} \Gamma_{3} V_{3}^{-1} D_{100}^{-1}\left(1+\Psi_{b}\right)\left(\frac{\phi_{b}^{2}}{20^{\circ}}\right)^{2} \text { yrs. }
$$

This corresponds to $\tau_{10}=0.1$ in eq. (28), suggesting that magnetically-powered, orbiting bursters might cease GRB activity too early to make a substantial contribution to the observable burst distribution $\left(\Psi_{b} \ll 1\right)$. However, accretion-powered bursters $(\S 5.2)$ would not be subject to this bursting lifetime limit.

\section{Concluding Discussion}

\subsection{Summary}

In this paper we have considered models for GRBs from high-velocity neutron stars in the galactic halo. In particular, we compared GRB observations with a model having the following properties: [1] bursters are born at positions distributed like young Pop. I stars in the galactic disk; [2] with randomly directed recoils $V_{r}=1000 V_{3} \mathrm{~km} \mathrm{~s}^{-1}$; [3] they emit GRBs at a constant rate, with [4] constant luminosity, and [5] the gamma ray emission is beamed parallel and anti-parallel to $\mathbf{V}_{\mathbf{r}}$, within an angular radius $\phi_{b}$.

This "standard halo beaming model" (S-HBM) adequately fits all published data on the distributions of GRBs from the BATSE and PVO experiments, when $12^{\circ} V_{3}^{-1}<\phi_{b}<30^{\circ}$. Models for GRBs from sources at cosmological distances fit present BATSE observations 
better than the S-HBM does, but not by a statistically-compelling margin. These fits to the data, for $V_{r}=1000 \mathrm{~km} \mathrm{~s}^{-1}$ and $\phi_{b}=20^{\circ}$, are shown in Figure 4 and Figure 6. Note that the fit improves for $V_{r}>1000 \mathrm{~km} \mathrm{~s}^{-1}$ (as found in most SGRs; Appendix A).

An important feature of the HBM is that almost all bursters born in the Andromeda Galaxy (M31) or other nearby external galaxies are unobservable at Earth because of misdirected beaming (LDT; Li, Fenimore and Liang 1996). Only when the sampling depth of the instrument reaches M31 can the effects of M31 possibly become evident. This means that the often-quoted upper bounds on the BATSE sampling depth based on considerations of M31 (e.g., LD92; Hakkila et al. 1994) do not apply to the HBM. Bounds on halo models based on the nondetection of X-ray bursts from M31 (Li \& Liang 1992) or in samples of nearby external galaxies also do not constrain the HBM, as long as the X-rays associated with GRBs are beamed in a similar way as the gamma rays.

We have also considered three refinements to the S-HBM, relaxing the assumptions [2], [4] and [5] in the above list:

Intensity variations across the beam. We analyzed models with centrally-peaked gamma-ray emission beams; i.e., with intensity declining as a function of angular separation from the beam axis, as in relativistic emission models. We found that the observable model results are not very sensitive to the details of the beam intensity distribution. Adequate fits to the data were obtained as long as the angular radius at which the intensity falls to half its central value is $\sim 20^{\circ}$, and the angle at which it drops by another factor $\sim 2$ is not many times larger.

Burst luminosity functions. We explored the consequences of a spread in GRB intrinsic brightnesses, considering luminosity functions of the form $\Phi(L) \propto L^{-\beta}$ for $L_{\min }<L<L_{\max }$. We found that, in the HBM as in other galactic halo models, only a limited dynamic range $L_{\max } / L_{\min } \leq 10$ or 20 is admissable among GRBs observed by BATSE and PVO. Depending 
on whether the power-law index satisfies $\beta<0$ or $\beta>0$, the spread in luminosities either improves or worsens the model's fit to the BATSE angular distribution. However, in either case the effect is small because the dynamic range is limited.

Models with a subpopulation of bound bursters. In the S-HBM, all bursters are born with recoil velocities $V_{r}=1000 V_{3} \mathrm{~km} \mathrm{~s}^{-1}$. Realistically, one expects bursters to aquire a distribution of $V_{r}$ values. The low $-V_{r}$ tail in this distribution could correspond to bursters that remain weakly-bound to the galaxy. Podsiadlowski, Rees \& Ruderman (1995) have shown that these bound stars follow non-periodic orbits in the non-spherical galactic halo potential, generally not returning to the small galactic radii where they were born. After one or more orbits, their magnetic pole (beaming axis) orientations are randomized. If they continue to emit (beamed) GRBs at late ages, then the bound and escaping bursters might produce comparable numbers of observable GRBs. (The exact ratio, eq. [28], depends upon several uncertain model parameters.) The observable distribution of GRBs would then be an appropriately weighted sum of the S-HBM burst distribution, describing the unbound bursters (Fig. 4 and Fig. 6), and the bound burster distribution studied by PRR. The resultant model angular distribution might be significantly more isotropic than that of the standard HBM, if the orbiting bursters are only weakly bound to the galaxy (see $\S 6.2$ ). This plausible refinement of the standard HBM merits further study.

\subsection{Conclusion: Cosmological or Galactic GRBs?}

Our main conclusion is as follows. The models studied in this paper give acceptable fits to observations as long as certain special conditions (e.g., uniform bursting rate, $\left.12^{\circ} V_{3}^{-1}<\phi_{b}<30^{\circ}\right)$ are satisfied. These conditions, although possible and arguably

plausible, have no clear and compelling theoretical justification. Other special conditions of varying plausibility are required in other halo models (e.g., LD92; see Hartmann et al. 1994 
for a comprehensive review of halo models). The cosmological hypothesis, on the other hand, fits present observations generically, and thus is much more attractive in this sense. Moreover present data are generally better fitted by the cosmological models than by the S-HBM, as shown by Fig. 6.

Based upon this powerful argument, we believe cosmological GRB models to be more promising than galactic halo models, given the evidence available at the time of writing. However, some caution is appropriate because there exists no compelling theoretical understanding of GRBs at either distance scale. The study of GRBs has taken many unexpected twists and turns in the past: only five years ago there was a wide consensus favoring neutron stars at distances $\lesssim 10^{2}$ pc.

If the cosmological hypothesis is correct, then continued BATSE data collection should be able to rule out the standard HBM using the test of Fig. 6. A doubling of the data base beyond the $3 \mathrm{~B}$ catalog might rule out the $\mathrm{S}-\mathrm{HBM}$ with $>3-\sigma$ confidence.

A plausible refinement of the standard HBM that might be more difficult to falsify includes some bursters that are weakly-bound to the galaxy (§6.3). Such a model can be jointly constrained by limits on GRBs - or associated X-ray bursts - from nearby external galaxies (e.g., Li \& Liang 1992), which limit the bound burster population, and BATSE angular position observations, which more strongly limit the unbound ones. We will quantify these constraints in a future study.

If GRB do come from the galactic halo, then deviations from isotropy on the sky will eventually manifest themselves. In any case, continued operation of BATSE is essential for making these tests. Checking BATSE with an independent experiment is also desirable. It is important to verify that systematic errors do not wash out the very low-level anisotropies predicted by halo models. 
GRB repetitions, if verified, could lend credence to halo models; however the predicted mean time interval between repeat bursts from old SGRs in the halo is $\tau_{G R B} \sim 100$ years, with some dependence on uncertain model parameters (eq. [10] and eq. [30]), so repeat events may be difficult to detect unless they are strongly clustered in time.⿴囗十

The durations of BATSE bursts were found to be anti-correlated with their intensities by Norris et al. (1993) [but see Mitrofanov et al. 1996]. This could be due to cosmological time dilation. However, this correlation, if verified, does not give strong evidence against the HBM. In the HBM more distant burst sources are systematically older, so any significant trend of increasing burst duration or spectral softening with age could mimic cosmological time dilation.

What are the strongest arguments in favor of galactic halo GRB sources? There is strong empirical evidence that a large number of old soft gamma repeaters exist in the extended galactic halo (DT92). We estimate the number of aged SGRs within $100 \mathrm{kpc}$ to be between $\sim 10^{4}$ and $\sim 10^{7}$, depending on values of the scaling parameters in equations

\footnotetext{
${ }^{18}$ Magnetically-powered GRBs would tend to cluster in time, like stellar flares, so incidents of GRB repetition should eventually be observed if the bursters are isolated magnetars. This could be an important observational diagnostic, because most cosmological GRB models do not allow burst repetitions.

19 Many bursts in the BATSE catalog show distinct episodes of bursting activity separated by $\sim 10-100 \mathrm{~s}$ intervals containing no detected emissions (Lingenfelter, Wang \& Higdon 1994). It would be interesting to search for such recurrences on somewhat longer timescales, $\sim 10^{2} \mathrm{~s}$ to $10^{4} \mathrm{~s}$, in order to place bounds on how long the time window for recurrence lasts. Unfortunately, BATSE is biased against recording data during a readout period lasting from $\sim 100$ s to $\sim 90$ minutes following any burst, when it it downloading data. A large fraction of bursters could produce lower-intensity recurrences during this period without any detections.
} 
(9) and (26). These halo stars have many properties required of classic GRB sources, as first pointed out by DT92. They are clearly distinct from radio pulsars (footnote 12 and Appendix A), thus avoiding the problem of over-concentration of bursters toward the galactic disk which plagues many theories based on neutron stars. Moreover, of all known astrophysical objects, SGRs have produced the emissions which most closely resemble GRBs. They have produced at least one burst of photons with a classic GRB-like spectrum (on March 5, 1979; FKL); and when they are young (ages $\lesssim 10^{4}$ yrs), they emit large numbers of softer-spectrum bursts with energies $\sim 10^{41}$ ergs, comparable to the energies of GRBs in the halo.

We have briefly outlined some candidate physical models for these bursters $(\S 2.3, \S 2.4$, and $§ 5)$. One promising class of models involves neutron stars with ultra-strong magnetic fields $B>10^{14} \mathrm{G}$, much stronger than the fields known to exist in ordinary radiopulsar magnetospheres. Such strong magnetic fields could provide the energy for flare-like halo GRBs (§5.1), or they could mediate the conversion of accretion energy into gamma rays (§5.2). Fields this strong could also drive crust fracture events in young neutron stars, producing SGR activity (TD95). [Note that the distributions of SGR burst energies and waiting times, and their time-correlations, are consistent with crust fractures; Cheng et al. 1996.] Finally, strong magnetic fields can drive neutrino emission anisotropies in newborn neutron stars, imparting recoils that propell the stars into the galactic halo (§2.4). Note that all of these mechanisms could conceivably operate in a non-HBM context, i.e., a halo GRB model in which the bursts are unbeamed or beamed in random directions (see footnote 14).

If aged SGRs do not emit classic GRBs, then it is still likely that these stars populate the galactic halo (DT92). If they are isolated magnetars, then they will rotate very slowly after leaving the galactic disk. They will cool rapidly once their magnetic activity ceases, 
becoming dark halo relics that are difficult to detect.

Several promising models of cosmological GRBs also involve ultra-strong magnetic fields (Thompson 1994 and references therein). As a general result, gravity-powered MHD flows in compact neutron objects can create fields of strength $B^{2} / 8 \pi \sim \epsilon G M^{2} / R^{4}$, where $\epsilon$ is the efficiency. This implies

$$
B \sim 10^{18}\left(\frac{\epsilon}{0.1}\right)^{1 / 2}\left(\frac{M}{M_{\odot}}\right)\left(\frac{R}{10 \mathrm{~km}}\right)^{-2} \mathrm{G} .
$$

Although strong by most standards, this is vastly below the fundamental limit of $\sim 10^{49}-10^{53}$ $\mathrm{G}$ at which magnetic fields become unstable to the vacuum creation of GUT-scale or Planck-scale monopoles.

Thus, the $\sim 10^{12} \mathrm{G}$ dipole fields of radiopulsars are a million times weaker than the strongest possible flux densities attainable, however briefly, in neutron star formation and coelescence. The mystery of gamma-ray bursts may require us to finally recognize and understand this new regime of strong magnetism.

We thank Chris Thompson for key contributions to the basic idea of the HBM, and Edward Fenimore for assistance with fitting the PVO data, and for many useful discussions. We also thank an anonymous referee for perceptive remarks which greatly improved our discussion of bound halo bursters. This research was supported by the NASA Theoretical Astrophysics Program, Grant No. NAG5-2773, and by the Texas Advanced Research Program, Grant No. ARP-279. HL gratefully acknowledges the support of the Director's Postdoctoral Fellowship at Los Alamos National Laboratory. 


\section{A. Observations of Soft Gamma Repeaters and Evidence for Recoils}

We have suggested that old SGRs in our galaxy are the sources of classic GRBs. This requires that SGRs aquire sufficiently large recoils to propell them into the galactic halo. Here we briefly review the evidence for large recoils in SGRs, and discuss the related issue of whether SGRs have normal stellar companions. We also briefly discuss how SGR observations might be accounted for in the magnetar model (which predicts large recoils, $\S 2.4)$.

SGR 0526-66 The error box for the 1979 March 5 event (Cline et al. 1982) and the SGR-like bursts which followed in its aftermath (Golenetskii, Ilyinski \& Mazets 1984) lie within supernova remnant N49 in the Large Magellanic Cloud. A point-like X-ray sourcę inside this error box (Rothschild, Kulkarni \& Lingenfelter 1994) may have a thermal spectrum, since no hard spectral component has been detected (Murakami 1996; Marsden et al. 1996). Its intensity is consistent with thermal emission from a magnetically-heated neutron star (TD96). If the SGR was born at the center of the supernova that produced $\mathrm{N} 49$, then the displacement of the X-ray source from the remnant center implies a transverse velocity (DT92)

$$
V_{\perp}=1200 \pm 240 \mathrm{~km} \mathrm{~s}^{-1}
$$

where we have used the estimated age of $\mathrm{N} 49, t_{\mathrm{N} 49} \approx 5400 \mathrm{yrs}$ (Vancura et al. 1992). The statistically expected $3-\mathrm{D}$ recoil velocity is larger by a factor $(3 / 2)^{1 / 2}$, or $\bar{V}=1500 \mathrm{~km} \mathrm{~s}^{-1}$.

No radio, infrared or optical emissions have been detected from SGR0526-66 down to faint limits (Dickel et al. 1995; Marsden et al. 1996). This is not surprizing since a neutron star born with the velocity of eq. (A1) could not remain bound in a binary (DT92); and

\footnotetext{
20 That is, a source with an observed X-ray image that is statistically consistent with the instrumental point-spread function.
} 
a neutron star with an 8.0 second rotation period, as clearly shown by the 1979 March 5 event (Mazets et al. 1979), could not produce a significant rotation-powered plerion.

SGR 1900+14 This burster has been localized to a position lying just outside the edge of the galactic supernova remnant G42.8+0.6 (Hurley et al. 1994). Like SGR 0526-66, it has a candidate point-like X-ray counterpart, just outside the burst error box (Hurley et al. 1996). Assuming that the SGR was at the center of the supernova that produced remnant G42.8+0.6, one finds (Hurley et al. 1996)

$$
V_{\perp}=1700\left(\frac{D}{5 \mathrm{kpc}}\right)\left(\frac{t_{\mathrm{SNR}}}{10^{4} \mathrm{yr}}\right)^{-1} \mathrm{~km} \mathrm{~s}^{-1}
$$

where $D$ is the distance from Earth, and $t_{\mathrm{SNR}}$ is the age of the supernova remnant (SNR). This implies $\bar{V} \sim 2100 \mathrm{~km} \mathrm{~s}^{-1}$.

A double infrared source, probably a bound pair of M5 supergiant stars which is heavily reddened by interstellar dust, has been suggested as a counterpart object to SGR 1900+14 (Vrba et al. 1996). This object lies 18" from the center of the observed X-ray image (Hurley et al. 1996) and 50" from the burst error box (Vrba et al. 1996) in a region of dense galactic starfields, so the identification is not certain. If the infrared binary is a true counterpart to the SGR, then the association of the SGR with the supernova remnant is almost certainly spurious, because no known process could impart a recoil of magnitude (A2) to a pair of M5 supergiant stars. However, the other SGRs are unambiguously associated with $\sim 10^{4}$-year-old SNRs. Thus we favor the interpretation that the infrared binary is not physically associated, and the SNR is. In this case, SGR 1900+14 closely resembles SGR 0526-66 in all its known (observed) properties.

SGR 1806-20 This burster is located within the $\sim 10^{4}$-year-old SNR G10.0-0.3 (Kulkarini et al. 1994). It also has an X-ray counterpart (Murakami et al. 1994) which is point-like, but with a measured non-thermal spectral component (Sonobe et al. 1994), unlike 
the other SGRs. Furthermore, there is a synchrotron radio plerion surrounding the SGR (Kulkarni et al. 1994) indicating that the burster drives an outflow of relativistic particles. The radio spectral index of the plerion is steeper than that of known rotation-powered pulsar plerions (Kulkarni et al. 1994).

In the magnetar model, this plerion could be powered by SGR bursts, as suggested by Kulkarni et al. (1994) and studied by Tavani (1994) and Harding (1996). Another possibility is that the radio nebula and non-thermal X-rays are powered by quasi-continuous low-amplitude Alfvén wave emissions from magnetically-induced deep crustal fractures (TD95). We favor this second interpretation, for reasons explained in TD96. The other SGRs do not power radio plerions; this might mean that the deep crustal field in other SGRs is strong enough to overwhelm lattice stresses, driving plastic creep rather than quasi-steady low-amplitude seismic activity (TD96).

Thus in our favored interpretation, SGR 1806-20 has the weakest magnetic flux density in the deep crust of known SGRs. Alternatively, it could have the most spatially disordered magnetic field in the deep crust, which would also drive a higher rate of crustal fractures. In either case, we would expect it to also have the lowest recoil velocity according to the mechanisms of $\S 2.4$. The displacement of SGR 1806-20 from the center of the SNR is difficult to estimate because of the irregular shape of the remnant and because of late energy injection into the plerion, but Kulkarni et al. (1994) roughly estimate $V_{\perp} \gtrsim 500 \mathrm{~km}$ $\mathrm{s}^{-1}$.

This velocity is small enough, and uncertain enough, that the burster could have remained bound to a companion if it formed in a tight and massive binary. In fact, a massive companion star has been detected (Kulkarni et al. 1995). It seems to be a heavily-reddened OB supergiant (Kerkwijk et al. 1995). Its physical association with the SGR is in little doubt since it coincides with the finely-measured VLA position of the plerion's central peak 
(Vasisht, Frail \& Kulkarni 1995).

Since 1806-20 is the only SGR with a confirmed companion and a radio plerion, it is tempting to speculate that the two phenomena are related, and that the plerion is accretion-driven. However, SGR 1806-20 has few properties in common with known accretion-powered radio nebulae (Vasisht, Frail \& Kulkarni 1995). Moreover, the X-ray power is much less than the required power in relativistic particles (TD96). Accretionpowered outflows from neutron stars (e.g., jets) can have only a modest outflow efficiency, so the ratio would be the other way around (TD95; TD96). Still, one should keep in mind that SGR 1806-20 is much more complex than the other two SGRs. It is possible that interaction with, or accretion from, its hot supergiant companion may play some role in this system, even if the SGR is a magnetar. More observations are needed.

If classic GRBs are produced by old halo SGRs, then bursters which remain in binaries, like SGR 1806-20, are constrained to not produce too many observable classic GRBs as they grow old. If they did, the concentration of GRBs toward the galactic plane could violate BATSE limits. In the HBM, this requirement is actually not very restrictive, because bursters in binaries have effectively random beaming directions, greatly reducing their observable burst rates compared to bursters which become unbound. Furthermore, GRBs from old SGRs in binaries might be suppressed by the environmental influences of their companions, or because values of $V_{r}$ that are sufficiently small to avoid binary disruption only occur - via the mechanism of $§ 2.4$ - in stars with systematically weaker and/or more disordered magnetic fields, which penetrate the neutron star core less deeply. Such stars would be expected to dissipate their smaller resevoirs of magnetic energy relatively quickly, showing less magnetic activity (GRBs) late in their lives. The fact that the detectable energy loss from SGR 1806-20 exceeds that of the other SGRs is consistent with this conjecture. 


\section{B. Alfvén Wave-Mediated GRBs in Halo Models}

The amplitude $\delta B$ of Alfvén waves required to to carry a luminosity of $L_{\gamma}=10^{41} L_{41}\left(\Delta \Omega_{\gamma} / 4 \pi\right) \operatorname{erg~s}^{-1}$, as appropriate for halo GRBs (cf. eq. [11]), is

$$
\delta B \sim\left(\frac{4 \pi L_{\gamma}}{c R^{2} \Delta \Omega_{A}}\right)^{1 / 2} \sim 7 \times 10^{9} L_{41}^{1 / 2}\left(\frac{\Delta \Omega_{\gamma}}{\Delta \Omega_{A}}\right)^{1 / 2} \mathrm{G}
$$

near the neutron star surface (e.g., Blaes et al. 1989, hereafter BBGM). In this equation $\Delta \Omega_{\gamma}=2 \pi\left(1-\cos \phi_{b}\right)$ is the solid angle of GRB emissions, and $\Delta \Omega_{A}$ is the solid angle of the neutron star surface through which Alfvén waves pass. These Alfvén waves presumably couple to shear waves in the crust, which have velocity $V_{\mu}=(\mu / \rho)^{1 / 2}$, where the shear modulus at density $\rho=10^{11} \rho_{11} \mathrm{gm} \mathrm{cm}^{-3}$ is $\mu=8 \times 10^{28} \rho_{11}^{1.40} \mathrm{erg} \mathrm{cm}^{-3}$, valid below neutron drip, $\rho_{11}<4.6$. This value of $\mu(\rho)$ comes from eq. (11) of TD95 and a power-law fit to the equilibrium nuclear parameters in the Baym, Pethick \& Sutherland (1971) equation of state. For a neutron star with (uniform, vertical) magnetic flux density $B$, the coupling between Alfvén and seismic waves occurs at the density $\rho_{x}$ where $B=\left[4 \pi \mu\left(\rho_{x}\right)\right]^{1 / 2}$, or

$$
\rho_{x}=10^{11} B_{15}^{1.43} \mathrm{gm} \mathrm{cm}^{-3}
$$

Matching the seismic energy flux $F_{s}=\rho V_{\mu}^{3} \epsilon^{2}$, where $\epsilon$ is the wave strain amplitude deep in the crust, to the surface Alfvén energy flux $F_{A}=c(\delta B)^{2} / 4 \pi$ implies a strain amplitude at the coupling depth of

$$
\epsilon=\frac{\delta B}{B}\left(\frac{V_{\mu}\left(\rho_{x}\right)}{c}\right)^{-1 / 2} \sim 0.3 B_{12}^{-1.3} L_{41}^{1 / 2}\left(\frac{\Delta \Omega_{\gamma}}{\Delta \Omega_{A}}\right)^{1 / 2} .
$$

Since this ignores wave reflection, it should be considered a lower bound on $\epsilon$. This

exceeds the dynamical yield (fracturing) strain for $B \sim 10^{12} \mathrm{G}$. Thus waves with sufficient amplitude to mediate a halo GRB would crumble a radiopulsar's crust and thereby dissipate energy, probably producing an excess of X-rays. Only for fields that satisfy

$$
B>2 \times 10^{13} L_{41}^{0.38}\left(\frac{\epsilon_{\text {yield }}}{10^{-2}}\right)^{-0.77}\left(\frac{\Delta \Omega_{\gamma}}{\Delta \Omega_{A}}\right)^{0.38} \mathrm{G}
$$


is the vibration amplitude small enough to avoid pulverizing the crust. This condition is numerically similar to the magnetic energy requirement of equation (17), but conceptually distinct.

Note that when equation (B4) is satisfied, there are actually four distinct physical domains for the propagation of waves in the crust: (1) $\rho>\rho_{\text {drip }} \approx 4.6 \times 10^{11} \mathrm{gm} \mathrm{cm}^{-3}$. At this depth, the shear waves have amplitude that varies with density as $\epsilon \propto \rho^{0.18}$, based on a power-law fit to the Negele \& Vautherin (1973) equilibrium composition. (2) $\rho_{x}<\rho<\rho_{\text {drip }}$, where $\rho_{x}$ is given by eqn. (B2). Shear waves with $\epsilon \propto \rho^{-0.8}$.

$\rho_{\text {rel }}<\rho<\rho_{x}$, where $\rho_{\text {rel }} \equiv\left(B^{2} / 4 \pi c^{2}\right)=9 \times 10^{7} B_{15}^{2} \mathrm{gm} \mathrm{cm}^{-3}$. Non-relativistic Alfvén waves with $\epsilon \propto \rho^{0.25}$. (4) $\rho<\rho_{\text {rel }}$. Relativistic Alfvén waves with $\epsilon=\delta B / B=$ constant. Thus $\epsilon\left(\rho_{x}\right)$ as given by eqn. (B33) is the maximum strain in the crust above the neutron drip layer.21

Linear Alfvén waves in a neutron star magnetosphere are accompanied by oscillating currents, $\delta \mathbf{j}=(c / 4 \pi)(\nabla \times \delta \mathbf{B})-(1 / 4 \pi)(\partial \delta \mathbf{E} / \partial t)$. If the ambient plasma density is so low that the drift velocities needed to sustain $\delta \mathbf{j}$ exceed $c$, then the wave is charge-starved. This occurs at plasma densities less than

$$
n_{*} \simeq \frac{\nu(\delta B)}{2 e c}=1 \times 10^{11} L_{41}^{1 / 2} \nu_{3} \mathrm{~cm}^{-3}
$$

where $\nu=10^{3} \nu_{3} \mathrm{~Hz}$ is the wave frequency. [Note that BBGM predict a frequency spectrum peaked at $\nu \sim 10^{3} E_{41}^{-1 / 3} \mathrm{~Hz}$ for bursts triggered by crust fractures. This comes from the condition that $\nu^{-1}$ be comparable to the shear wave crossing-time of the fracture.] BBGM

\footnotetext{
${ }^{21}$ BBGM did a careful study of the coupling of seismic and Alfvén waves, including wave reflection effects. However, their numerical results for $B=10^{11} \mathrm{G}$ are in the regime in which zone (3) effectively does not exist (i.e., $\rho_{x}$ occurs at a depth that is less than one wavelength for Alfvén waves in the relevant frequency range).
} 
have speculated that charge-starved Alfvén waves accelerate charges until they are limited by radiation losses, thus producing GRBs as the waves undergo nonlinear damping. If this is true, then GRB emission from ordinary radiopulsars might occur only after they cross the "death line" at age $\sim 2 \times 10^{7} B_{12}^{-0.95}$ yrs (Chen \& Ruderman 1993), after which time the ambient plasma density in the magnetosphere is less than $n_{*}$. This could produce a delayed turn-on of GRBs from pulsars, as in the galactic halo model of Li \& Dermer (1992). However, the energy requirements for bursters (eq. [13] and eq. [17]), the crust-crumbling limit (eq. [B4]) and the over-concentration of old pulsars to the galactic disk are all potential difficulties with this scenario (LD92). Magnetars, which may avoid these drawbacks, cross the death line at $t_{D} \sim 2 \times 10^{5} B_{14}^{-1}$ yrs, comparable to the length of the SGR activity epoch. Subsequently, there exists a co-rotation charge density in the magnetosphere of magnitude (Goldreich \& Julian 1969)

$$
n_{G J} \simeq \frac{B}{P_{\text {rot }} e c} \simeq 1 \times 10^{12} B_{14}^{1 / 2}\left(\frac{t}{t_{D}}\right)^{-1 / 2} \mathrm{~cm}^{-3},
$$

where we have assumed a value of $P_{\text {rot }}(t)$ appropriate for spindown driven by a constant dipole field. Comparison with eq. (B5) shows that, just after crossing the death line, Alfvén waves carrying luminosities characteristic of halo GRBs are not charge-starved near a magnetar's surface. The waves become charge-starved only after a time period

$$
t_{*}=4 \times 10^{7} L_{41}^{-1} \nu_{3}^{-2} \mathrm{yrs},
$$

independent of $B$ in this high- $B$ domain. Interestingly, this is close to the turn-on time required by LD92. The connection of charge-starved Alfvén waves to GRBs is uncertain, and merits further study. 


\section{FIGURE CAPTIONS}

Fig. 1.- Schematic diagram of a beamed gamma-ray burster escaping from the Galaxy. The Galaxy is depicted in cross-section by a bulged disk, oriented horizontally at the bottom of the figure. The burster is born at point $\mathbf{A}$ in the galactic disk, with a recoil velocity $\mathbf{V}_{\mathbf{r}}$ in a random direction. Gamma ray emissions occur within indicated cones of angular radius $\phi_{b}$ about the star's magnetic axis $\mu$, which we assume is nearly aligned with $\mathbf{V}_{\mathbf{r}}$. As the star sails into the halo, reaching point $\mathbf{B}$ and beyond, the line of sight from Earth to the star gets increasingly aligned with $\mathbf{V}_{\mathbf{r}}$. Thus more and more escaping bursters become visible at larger distances $r$ from the galactic center. The fraction of all bursters which are detectable increases with distance as the transverse area of the beaming cone in the galaxy, or $\sim r^{2}$. This tends to counter-balance the free-streaming density trend, $n \propto r^{-2}$ within a "core radius" $R_{c} \sim R_{o} / \phi_{b}$, where $R_{o} \sim 10 \mathrm{kpc}$ is a typical galactic disk dimension. At distances larger than $R_{c}$, all bursters are detectable at Earth, and the $n \propto r^{-2}$ density trend prevails. Because only a tiny fraction $1-\cos \phi_{b} \simeq 0.06\left(\phi_{b} / 20^{\circ}\right)^{2}$ of all bursters are visible when they are born in the galactic disk, the observable dipole anisotropy of GRB positions, $\langle\cos \Theta\rangle$, is greatly reduced compared to what it would be if bursts were emitted isotropically. 
Fig. 2.- The 2-D cross section of the "zone of avoidance" (ZOA) for beaming angles $\phi_{b}=10^{\circ}, 20^{\circ}$ and $40^{\circ}$. The thick horizontal line represents the galactic plane where the galactic center $(\mathrm{GC})$ and Earth are located. The circles are loci within which bursting stars born at GC and moving in straight lines are not detectable at Earth. Everywhere within these ZOA circles, the angles between the star-to-Earth and star-to-GC directions are larger than $\phi_{b}$. A star becomes detectable either when it travels beyond the circle, or when it is inside the lens-shaped overlap region of circles directly between GC and Earth. The radius of the circles are $D_{\text {sun }} / \sin \phi_{b}$, where $D_{\text {sun }}=8.5 \mathrm{kpc}$.

Fig. 3.- Parmeters describing the spatial distribution of detectable bursters in the HBM are plotted as a function of distance from Earth, for several different beaming angles $\phi_{b}$ : $90^{\circ}$, or unbeamed (thick solid line); $40^{\circ}$ (thin solid line); $20^{\circ}$ (dotted); $10^{\circ}$ (dashed) and $5^{\circ}$ (alternating short and long-dashed). The thick long-dashed horizontal lines in each subplot are the expected values if the distribution is isotropic at all distances. Filled circles, placed at $D=180 \mathrm{kpc}$ in each subplot, are current BATSE values for 1122 bursts; the error bars show $3-\sigma$ statistical uncertainties. Models with $\phi_{b} \geq 40^{\circ}$ or $\phi_{b} \leq 10^{\circ}$ are evidently ruled out by current observational limits, but a significant range of BATSE sampling depths $D_{B} \gtrsim 100$ kpc are allowed if $\phi_{b} \sim 20^{\circ}$.

Fig. 4.- Comparison of the intensity distribution of the HBM with combined PVO and BATSE data. Note the homogeneous, Euclidean "core" with slope $-3 / 2$ at the bright end, which turns over to a significantly milder slope at the faint end. A beaming angle $\phi_{b}=20^{\circ}$ is used in the HBM plotted here, and the (standard-candle) intrinsic burst luminosity is varied to make the fitting, which has a $\chi^{2} \sim 6.9$ with 12 degrees of freedom. The implied peak luminosity, including the beaming reduction factor, is $\sim 1.5 \times 10^{40} \mathrm{ergs} \mathrm{s}^{-1}$. 
Fig. 5.- Comparison of the intensity distribution of HBM with BATSE only. Again, $\phi_{b}=20^{\circ}$ but the derived peak luminosity from this fitting is slightly smaller than that in Figure 4 .

Fig. 6.- Cumulative plots of the angular (an)isotropy measures versus peak photon flux $P_{256}$, for bursts in the $3 \mathrm{~B}$ catalog (814 in total). The solid curve is the HBM prediction for $V_{r}=1000 \mathrm{~km} \mathrm{~s}^{-1}$ and $\phi_{b}=20^{\circ}$; data points are from BATSE; the dashed line shows expected values if the bursts are distributed isotropically on the sky. The intrinsic burst peak luminosity found by fitting the $\log \mathcal{N}-\log P$ distribution was used to fix the the HBM curves' horizontal positions. Error bars on the BATSE points show 1- $\sigma$ statistical uncertainties appropriate for comparing the data with the HBM. Both models (HBM and isotropy) are corrected for BATSE's imperfect sky coverage. Isotropy gives a better fit to the data than does the HBM. However, the data are consistent at the $\lesssim 2-\sigma$ level or better with both models, at all observed flux levels.

Fig. 7.- Gamma-ray intensity, $I(\theta)$, is plotted as a function of angle $\theta$ away from the beam axis, for the relativistic beam model of Mao \& Yi (1994). In this model, luminous material is expanding uniformly into a cone of half opening-angle $\Delta \theta$ with Lorentz factor $\gamma$, and emitting photons isotropically in the material rest frame. In this plot we keep $\Delta \theta=20^{\circ}$, while varying $\gamma$ as shown in the figure legend. Also shown is the $\phi_{b}=20^{\circ}$ uniform beam case (solid line), which is equivalent to $\Delta \theta=20^{\circ}$ and $\gamma=\infty$.

Fig. 8. - The amounts by which observable GRB angular statistics in galactic halo models with variable beaming patterns $I(\theta)$ differ from their values in the uniform beam ("unibeam") HBM, $\phi_{b}=20^{\circ}$, are plotted as a function of peak photon flux. The beam intensity models considered here have $\Delta \theta=20^{\circ}$ and $\gamma=3,5,10$ as indicated in the figure legend; corresponding $I(\theta)$ functions are shown in Figure 7. The curves are not completely smooth because of the finite number of stars used in the Monte Carlo simulations. 
Fig. 9.- Similar to Figure 6 but including the effects of luminosity functions. Filled dots are from BATSE; $3-\sigma$ error bars are shown at the faint end. The thick solid line is for the HBM assuming mono-luminosity GRBs (standard candles). The broken lines are HBM results for two alternative luminosity functions discussed in the text: $\phi(L) \propto L^{-3}$ for $L_{\min }<L<L_{\max }$ with a dynamic range $L_{\max } / L_{\min }=20$ [dotted line]; and $\phi(L) \propto L^{3}$ with $L_{\max } / L_{\min }=4$ [dashed line]. It is clear that a GRB luminosity function with a positive (negative) slope tends to improve (worsen) the HBM fit to BATSE angular data. 


\section{REFERENCES}

Band, D. 1992, Ap. J. 400, L63.

Baring, M. G. 1993, ApJ, 418, 391

Baym, G., Pethick, C. \& Sutherland, P. 1971, ApJ, 170, 299

Bisnovatyi-Kogan, G.S. 1996, in High-Velocity Neutron Stars and Gamma-Ray Bursts, eds. R. Rothschild \& R.E. Lingenfelter (NY: AIP), 38

Blaes, O., Blandford, R., Goldreich, P. \& Madau, P. 1989, ApJ, 343, 839 (BBGM)

Briggs, M.S. 1993, Ap J, 407, 126

Briggs, M.S. et al. 1996, Ap J, 459, 40

Bulik, T. \& Lamb, D.Q. 1996, in Gamma-Ray Bursts: 3rd Huntsville Workshop, eds. C. Kouveliotou, M.S Briggs \& G.J. Fishman (NY: AIP), 345

Burrows, A. \& Hayes, J. 1996, in High-Velocity Neutron Stars and Gamma-Ray Bursts, eds. R. Rothschild \& R.E. Lingenfelter (NY: AIP), 25

Chen, K. Y. \& Ruderman, M. 1993, ApJ, 402, 264

Cheng, B., Epstein, R.I., Guyer, R.A. \& Young, A.C. 1996, Nature, 382, 518

Chugai, N.N. 1984, Sov. Astron. Lett., 10, 87.

Cline, T.L. et al. 1982, ApJ, 255, L45

Colgate, S.A. 1992, in Gamma-Ray Bursts: Observations, Analyses \& Theories, eds. Ho, C. et al. (Cambridge Univ. Press) p. 75 
Colgate, S.A. \& Leonard, P.J.T. 1994, in Gamma Ray Bursts, AIP Conf. Proc. 307, eds. G.J. Fishman et al., (AIP: New York) p. 581.

Colgate S.A. \& Petschek A.G., 1981, ApJ, 248, 771

Cordes, J.M. 1986, Ap. J. 311, 183.

Davis, L. \& Goldstein, M. 1970, ApJ, 159, L81

Dermer, C.D. 1990, Ap.J., 360, 197

Dickel, J.R. et al. 1995, Ap.J., 448, 623

Dorofeev, O.F., Rodionov, V.N. \& Ternov, I.M. 1985, Sov. Astron. Lett., 11, 123

Dubinski, J. 1992, Ap.J., 401, 422

Duncan, R. C., Li, H. \& Thompson, C. 1993, Compton Gamma Ray Observatory, eds. M. Friedlander et al. (NY: AIP), 1074 (DLT)

Duncan, R. C. \& Thompson, C. 1992, ApJ, 392, L9 (DT92)

Duncan, R. C. \& Thompson, C. 1996, in High-Velocity Neutron Stars and Gamma-Ray Bursts, eds. R. Rothschild \& R.E. Lingenfelter (NY: AIP), 111 (DT96)

Eichler, D., \& Silk, J. 1992, Science, 257, 937

Fabian, A.C. \& Podsiadlowski, P. 1993, MNRAS, 263, 49

Fatuzzo, M. \& Melia, F. 1993, Ap.J., 414, L89.

Fenimore, E. E., Klebesadel, R.W. \& Laros, J.G., 1996, ApJ, 460, 964 (FKL)

Fenimore, E. E. et al. 1993, Nature, 366, 40

Fenimore, E. E., Epstein, R. I., \& Ho, C. 1993, A\&AS, 97, 59 
Fishman, G.J., Meegan, C.A., Watts, J.W. \& Derrickson, J.H. 1978, ApJ, 223, L13

Flowers, E. and Ruderman, M.A. 1977, Ap. J., 215, 302.

Frail, D.A. 1996, in High-Velocity Neutron Stars and Gamma-Ray Bursts, eds. R. Rothschild \& R.E. Lingenfelter (NY: AIP), 3

Frail, D.A. 1997, in The Many Faces of Neutron Stars, eds. A. Alpar, R. Buccheri \& J. van Paradijs (NATO Advanced Study Institute) in press

Frail, D.A. \& Moffett, D.A. 1993, ApJ, 408, 637

Goldreich, P. 1970, ApJ, 160, L11

Goldreich, P. \& Julian, W.H. 1969, ApJ, 157, 869

Goldreich, P. \& Reisseneger, A. 1992, ApJ, 395, 250

Golenetskii S.V., Ilyinskii, V.N. \& Mazets, E.P. 1984, Nature, 307, 41

Hakkila, J. et al., 1994, ApJ, 422, 659

Hakkila, J. et al. 1995, ApJ, 454, 134

Harding, A. K. 1996, in High-Velocity Neutron Stars and Gamma-Ray Bursts, eds. R. Rothschild \& R.E. Lingenfelter (NY: AIP), 118

Harrison, E.R. \& Tadmaru, E. 1975, ApJ, 201, 447

Hartmann, D. 1992, Comm. Ap., 16, 231

Hartmann, D., Epstein, R.I. \& Woosley, S.E. 1990, ApJ, 348, 625

Hartmann, D.H. et al. 1994, ApJS, 90, 893

Hartmann, D. \& Narayan, R. 1996, Ap J, 464, 226 
Harwit M. \& Salpeter E.E., 1973, ApJ, 186, L37

Ho, C., Epstein, R.I. \& Fenimore E.E. 1990, ApJ, 348, L25

Horak, J.M., Hakkila, J., Emslie, A.G., \& Meegan, C.A. 1996, ApJ, 462, 131

Hurley, K., Sommer, M., Kouveliotou, C., Fishman, G., Meegan, C., Cline, T., Boer, N. \& Neil, M. 1994, ApJ, 431, L31

Hurley K. et al., 1994, ApJ, 431, L31

Hurley, K. et al. 1996, ApJ, 463, L13

Janka, H.-Th. \& Müller, E. 1994, A.A., 290, 496

Jennings, M. C., \& White, R.S. 1980, ApJ, 238, 110

Katz, J.I. 1996, ApJ, 463, 305

Koshut, T. M. et al. 1996, ApJ, 463, 570

Kouvelioutou, C. et al. 1993, ApJ, 413, L101

Krolik, J.H. \& Pier, E.A. 1991, Ap J, 373, 277

Kulkarni, S.R., Frail, D.A., Kassim, N.E., Murakami, T. \& Vasisht, G. 1994, Nature, 368, 129

Kulkarni, S.R. et al. 1995, Ap.J., 440, L61

Kusenko, A. \& Segrè, G. 1997, Phy. Rev. Lett., in press

Lamb, D. Q. 1995, PASP, 107, 1156

Lamb, D.Q., Bulik, T. \& Coppi, P.S. 1996, in High-Velocity Neutron Stars and Gamma-Ray Bursts, eds. R. Rothschild \& R.E. Lingenfelter (NY: AIP), 219 
Li, H. \& Dermer, C. 1992, Nature, 359, 514 (LD92)

Li, H. \& Duncan, R. C. 1996, in High-Velocity Neutron Stars and Gamma-Ray Bursts, eds. R. Rothschild \& R.E. Lingenfelter (NY: AIP), 244 (LD96a)

Li, H \& Duncan, R.C. 1996b, in Gamma-Ray Bursts: 3rd Huntsville Workshop, eds. C. Kouveliotou, M.S Briggs \& G.J. Fishman (NY: AIP), 366 (LD96b)

Li, H., Duncan, R. C. \& Thompson, C. 1994, in Gamma-Ray Bursts, eds. G. Fishman et al. (NY: AIP), 600 (LDT)

Li, H. \& Liang, E.P. 1992, ApJ, 400, L59

Li, H., Fenimore, E. E. \& Liang, E. P. 1996 ApJ, 461, L73

Liang, E.P. \& Li, H. 1993, A.A. 273, L53.

Lin, D., Woosley, S.E. \& Bodenheimer, P. 1991, Nature, 353, 857

Lingenfelter, R.E., Wang, V.C. \& Higdon, J.C. 1994, in Gamma-Ray Bursts, eds. G. Fishman et al. (NY: AIP), 222

Lu, E.T., Hamilton, R.J., McTiernan, J.M. \& Bromund, K.R. 1993, ApJ, 412, 841

Lubin, L.M. \& Wijers, R.A.M.J. 1993, ApJ, 418, L9

Lorimer, D.R., Lyne, A.G. \& Anderson, B. 1995, MNRAS, 275, L16.

Lyne, A. G. \& Lorimer, D. R. 1994, Nature, 369, 127

Mao, S. \& Yi, I. 1994, ApJ, 424, L131

Marsden, D., Rothschild, R.E., Lingenfelter, R.E. \& Puetter, R.C. 1996, ApJ, 470, 513.

Mazets, E.P. et al. 1979, Nature, 282, 587 
McKinnon, M.M. 1993, ApJ, 413, 317

Meegan, C.A. et al. 1992, Nature, 355, 143.

Meegan, C. A. et al. 1996, ApJS, 106, 65

Melia, F. \& Fatuzzo M. 1991, ApJ, 383, L57.

Michel, F. C. \& Goldwire, H. C. 1970, Astrophys. Lett., 51, 21

Miller, M.C. 1995, ApJ, 448, L29

Miyamoto, M. \& Nagai, R. 1975, Pub. Ast. Soc. Jap., 27, 533

Mitrofanov, I.G. et al. 1996, Ap.J. 459, 570

Murakami, T. 1996 in High-Velocity Neutron Stars and Gamma-Ray Bursts, eds. R. Rothschild \& R.E. Lingenfelter (NY: AIP), 67

Murakami T., et al., 1994, Nature, 368, 127

Murphy, R.J. et al. 1993 in Compton Gamma Ray Observatory eds. M. Friedlander, N. Gehrels \& D.J. Macomb (NY: AIP) p. 619.

Nagele, J.W. \& Vautherin, D. 1973, Nuc. Phys. A207, 298

Norris, J.P, Hertz, P., Wood, K.S. \& Kouveliotou, C. 1991, ApJ, 366, 240

Norris J.P. et al. 1993, Ap.J. 424, 540

Paczyński, B. 1990, Ap. J., 348, 485

Paczyński, B. 1991, Acta. Astron., 41, 157

Paczyński, B. 1995, PASP, 107, 1176 
Petrosian, V. 1993, ApJ, 402, L33

Petrosian, V. \& Lee, T.T. 1996, ApJ, 467, L29

Piran, T., \& Shemi, A. 1993, ApJ, 403, L67

Podsiadlowski, P., Rees, M. \& Ruderman, M. 1995, MNRAS, 273, 755 (PRR)

Ramaty, R. \& Mandzhavidze, N. 1993, in Compton Gamma Ray Observatory eds. M. Friedlander, N. Gehrels \& D.J. Macomb (NY: AIP) p. 643.

Riffert, H., Mészáros, P. \& Bagoly, Z. 1989, ApJ, 340, 443

Rothschild, R.E., Kulkarni, S.R. \& Lingenfelter, R.E. 1994, Nature, 368, 432

Ruderman, M. 1991, Ap.J., 382, 576.

Salpeter, E.E. \& Wasserman, I.M. 1993, in Planets Around Pulsars, eds. J.A. Phillips, S.E. Thornsett \& S.R. Kulkarni, 345

Schmidt, M. 1968, Ap.J., 151, 393

Schmidt, M., Higdon, J.C. \& Hueter, G. 1988, Ap.J., 329, L85

Shklovskii, I.S. \& Mitrofanov, I.G. 1985, MNRAS, 212, 545

Shimizu, T., Yamada, S. \& Sato, K. 1994, Ap.J., 434, 268

Simon, T. 1990, Ap.J. 359, L51

Sonobe, T., Murakami, T., Kulkarni, S.R., Aoki, T. \& Yoshida, A. 1994, ApJ, 436, L23

Stern, B.E. \& Svensson, R. 1996, Ap.J., 469, L109

Sturrock, P.A. 1986, Nature, 321, 47 
Tavani, M. 1994, ApJ, 431, L83

Tegmark, M., Hartmann, D.H., Briggs, M.S. \& Meegan, C.A. 1996, ApJ, 468, 214

Thompson, C. 1994, MNRAS, 270, 480

Thompson, C., \& Duncan, R.C 1993, ApJ, 408, 194 (TD93)

Thompson C. \& Duncan R.C., 1994, in Backer D., Fruchtner A.S., Tavani M., eds., Proc. Workshop on Millisecond Pulsars, Aspen, Colorado (Astron. Soc. Pac., San Francisco), 303 (TD94)

Thompson, C., \& Duncan, R.C 1995, MNRAS, 275, 255 (TD95)

Thompson, C., \& Duncan, R.C 1996, ApJ, 473, 322 (TD96)

Tremaine S. \& Żytkow A.N., 1987, ApJ, 301, 155

van Buren, D. 1981, ApJ, 249, 297

van der Kruit, P. C. 1987, in The Galaxy, Series C: Mathematical and Physical Sciences, Vol. 207, ed. G. Gilmore and R. Carswell (Dordrecht: Reidel)

van Kerkwijk, M.H., Kulkarni, S.R., Matthews, K. \& Neugebauer, G. 1995, ApJ, 444, L33

Vasisht, G., Frail, D.A. \& Kulkarni, S.R. 1995, ApJ, 440, L65

Vrba, F.J. et al. 1996, ApJ, 468, 225

Wasserman, I. \& Salpeter, E.E. 1994, ApJ., 433, 670

Woosley, S.E. \& Herant M. 1996, in High-Velocity Neutron Stars and Gamma-Ray Bursts, eds. R. Rothschild \& R.E. Lingenfelter (NY: AIP), 263

Ulmer, A., Wijers, R.A.M.J. \& Fenimore E.E. 1995, ApJ, 440, L9 (UWF) 
Vancura O., Blair W.P., Long K.S. \& Raymond J.C., 1992, ApJ, 394, 158

Vasisht G., Kulkarni S.R., Frail D.A. \& Greiner J., 1994, ApJ, 431, L35

Vilenkin, A. 1995, Ap.J., 541, 700

Yi, I. 1993, Phys. Rev. D, 48, 4518

Yi, I. \& Mao, S. 1994, Phys. Rev. Lett., 72, 3750 


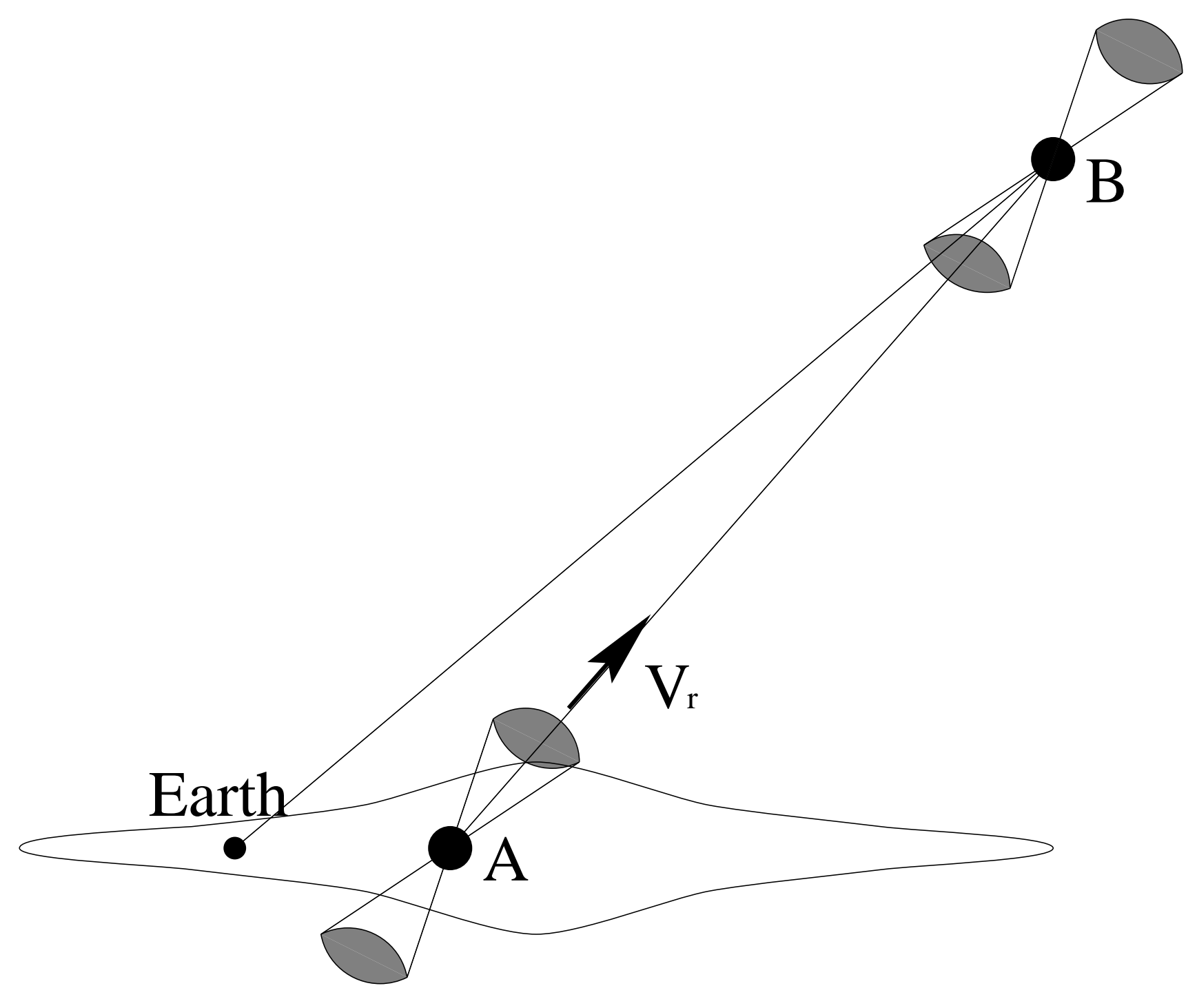




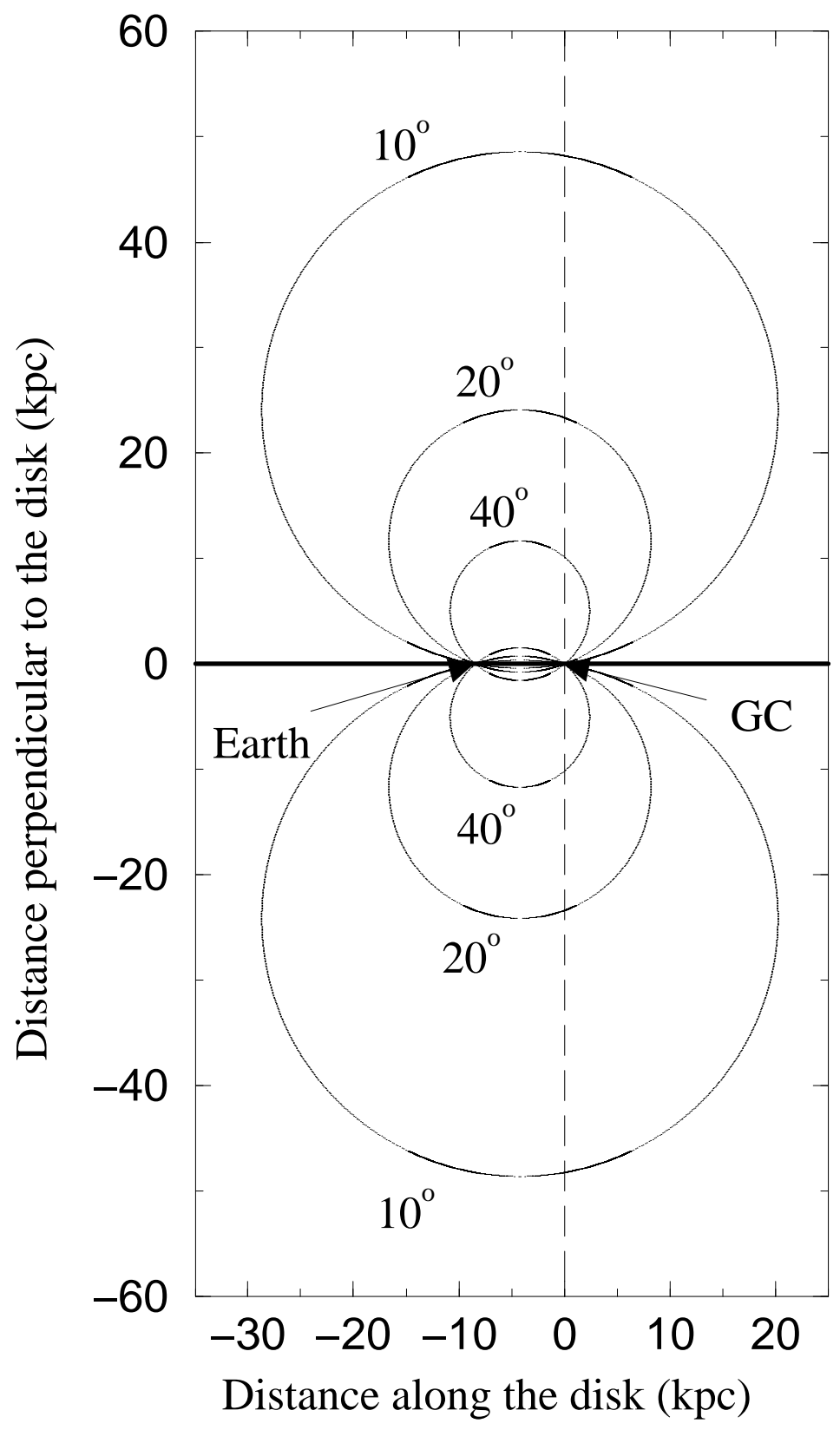




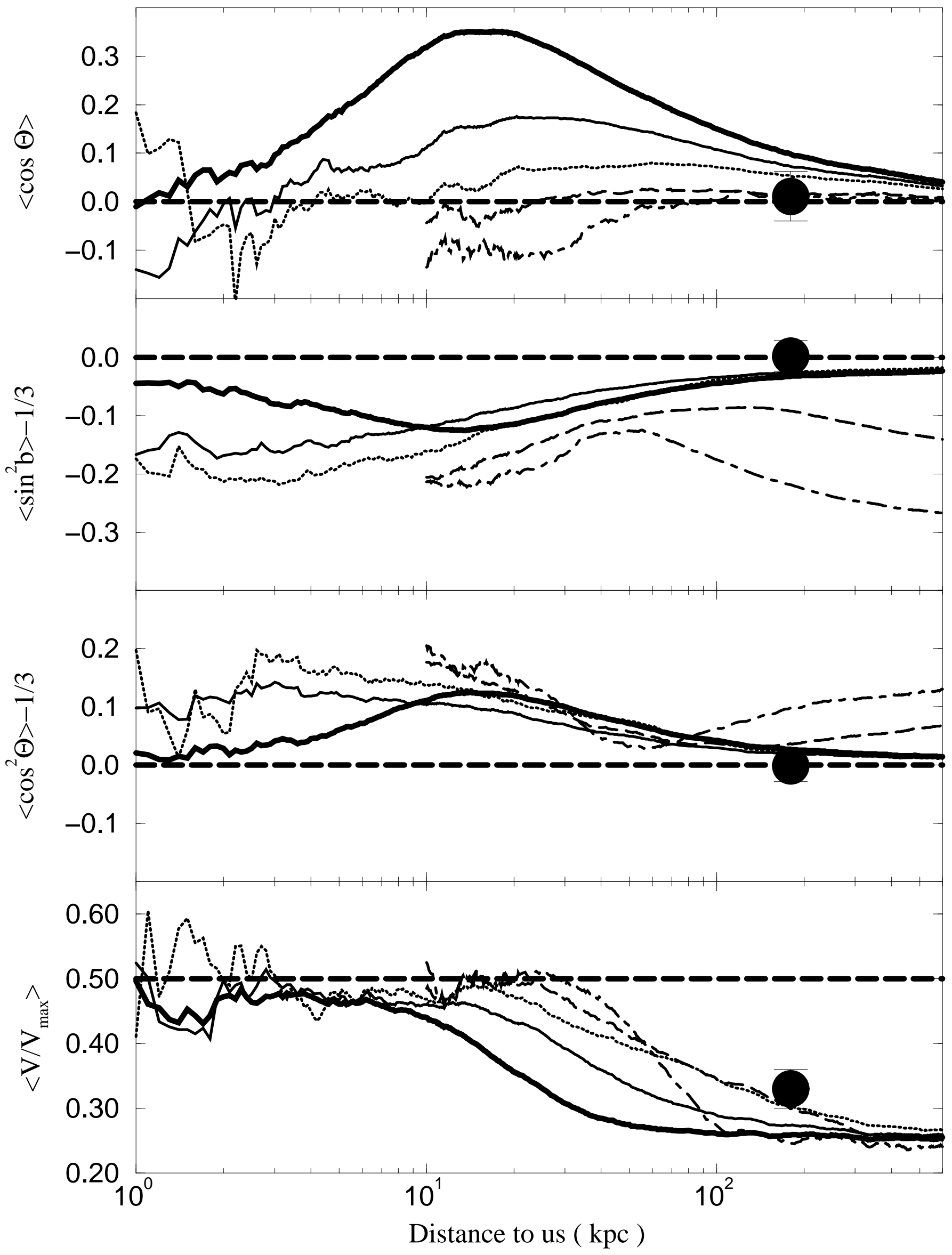




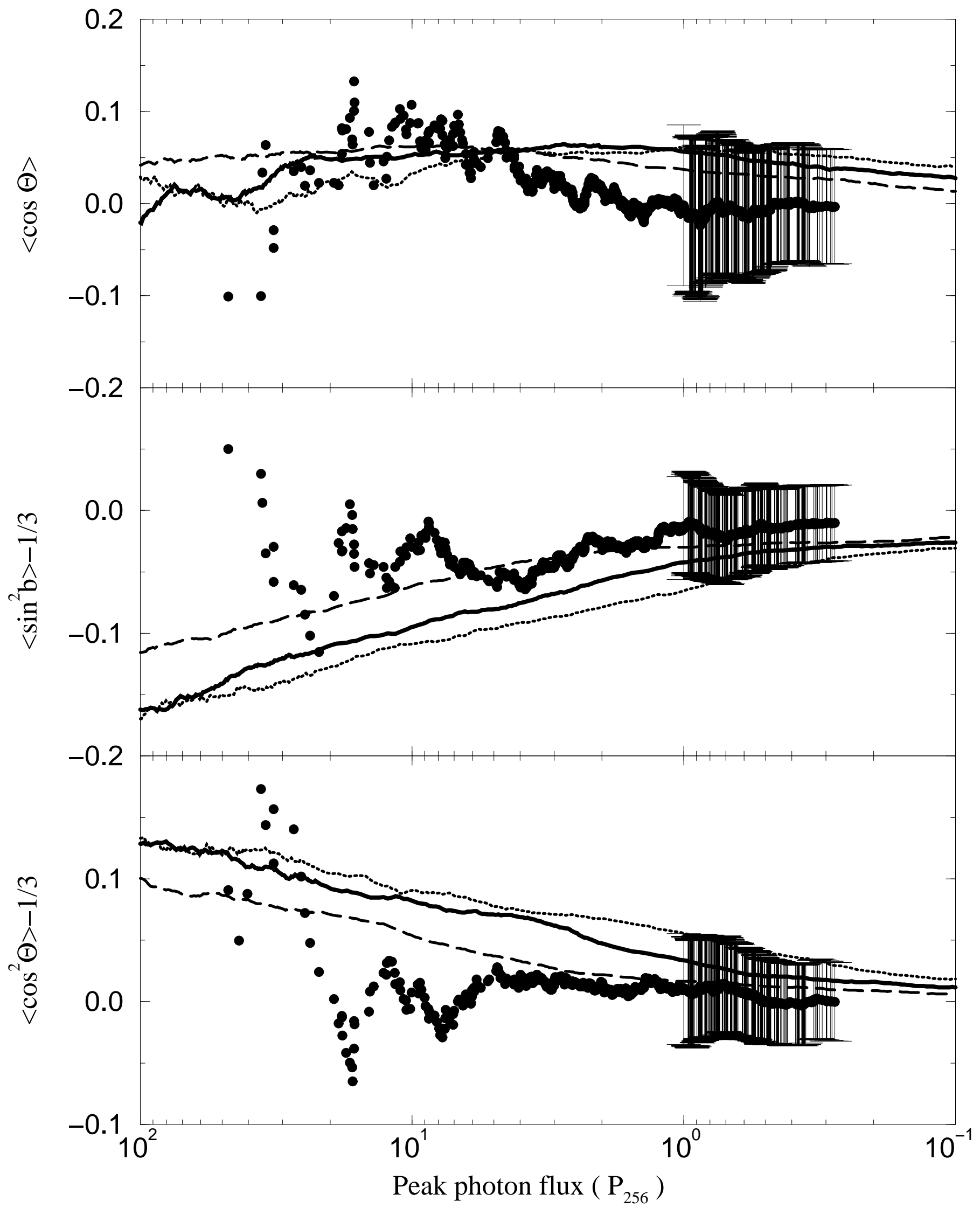




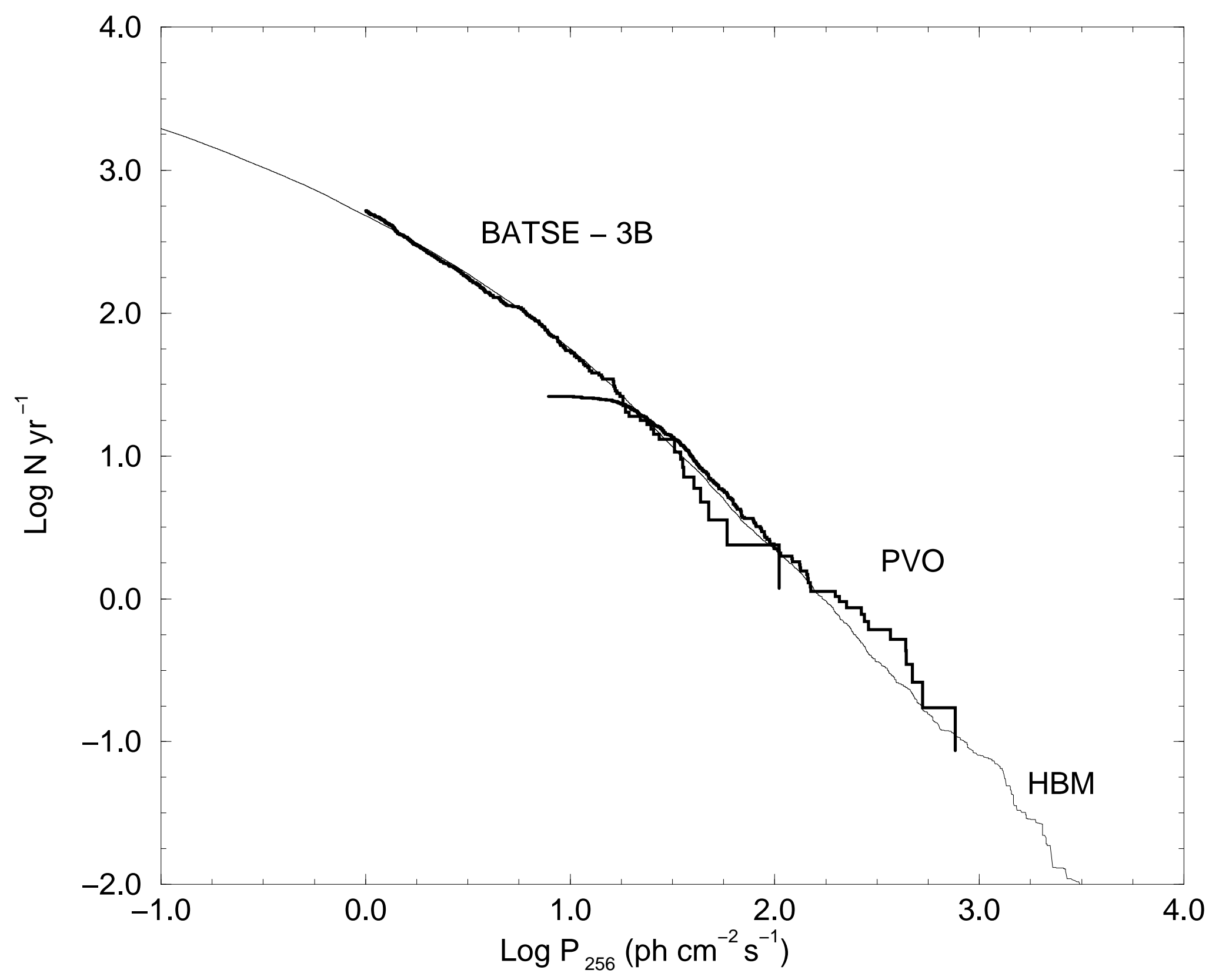




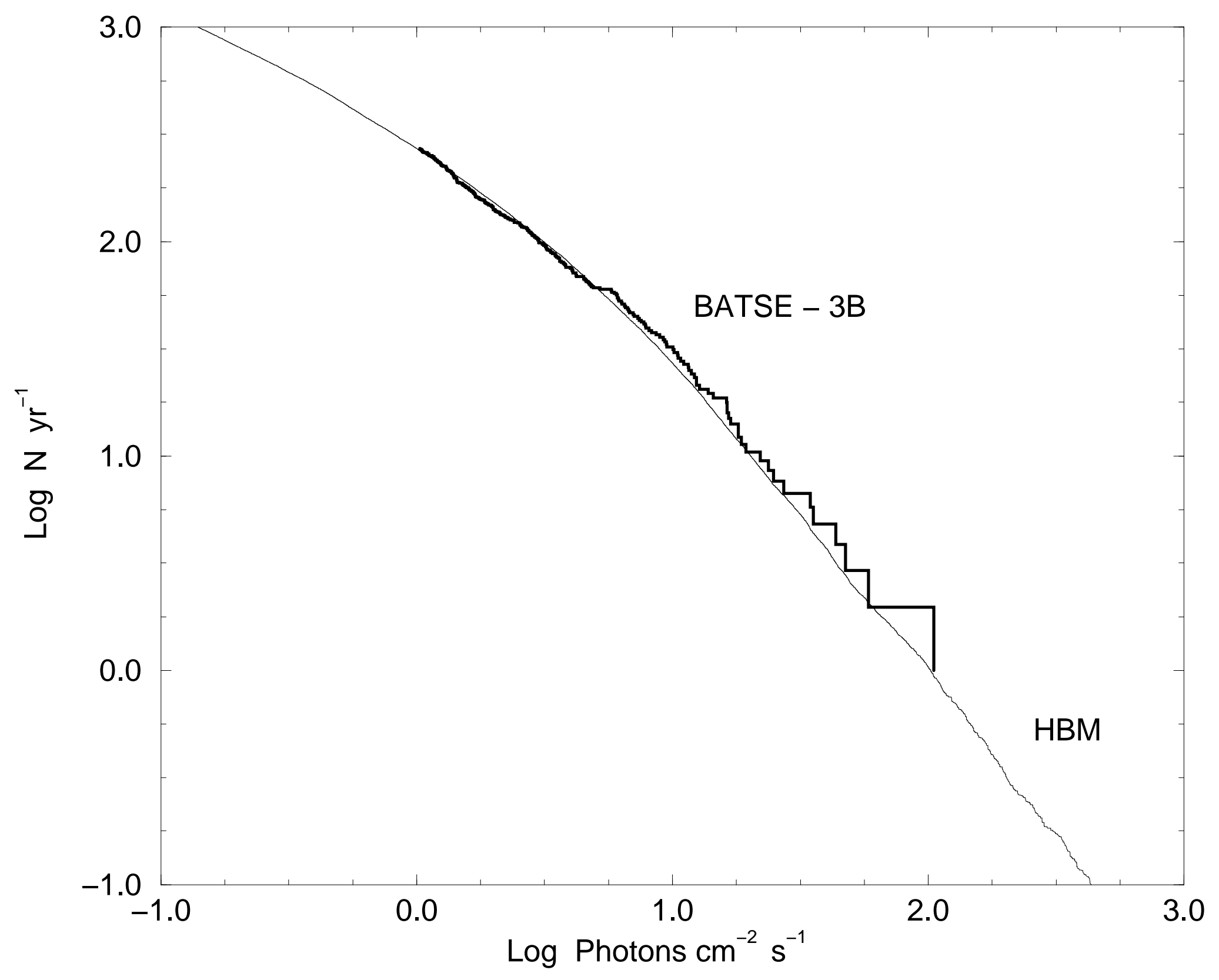




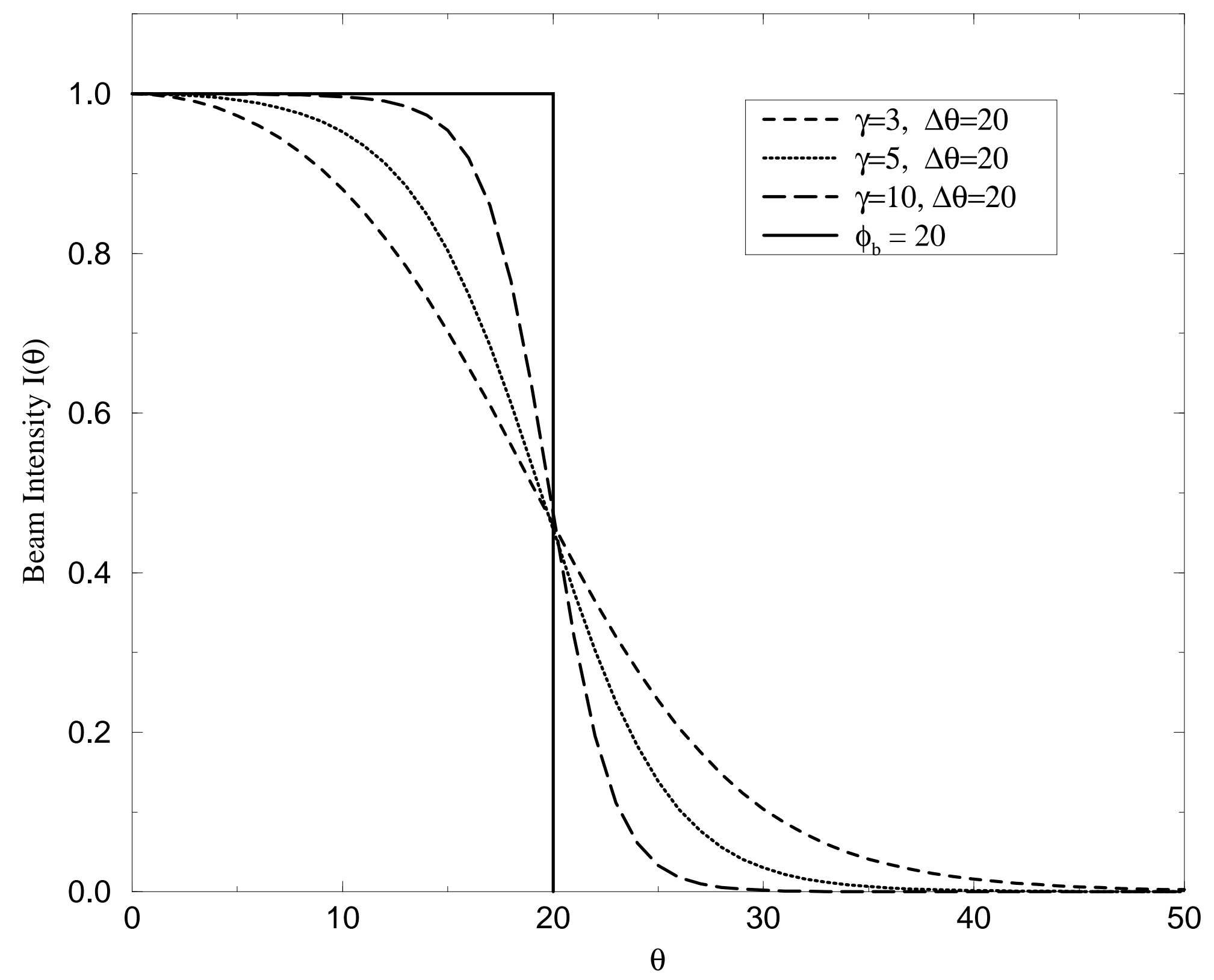




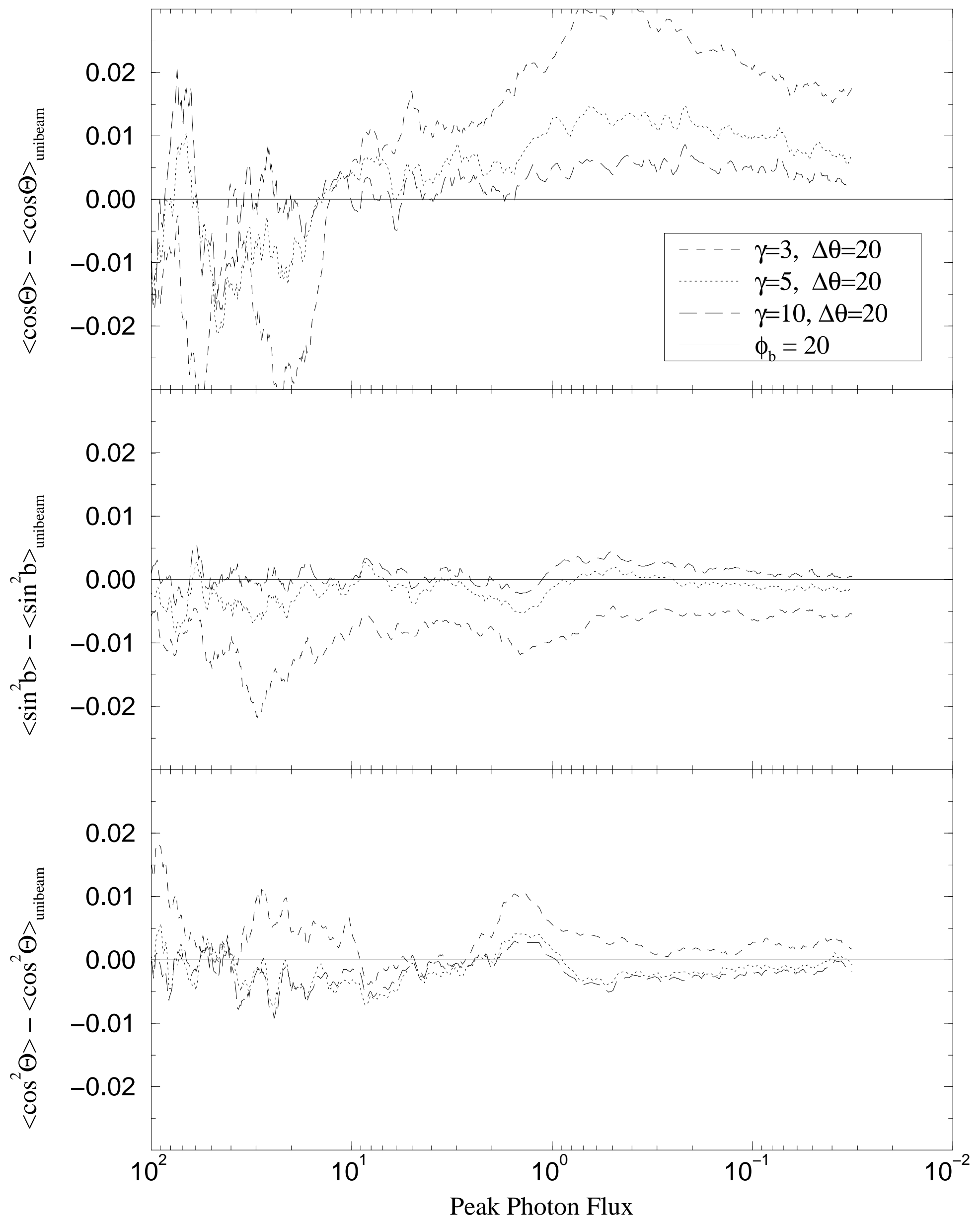




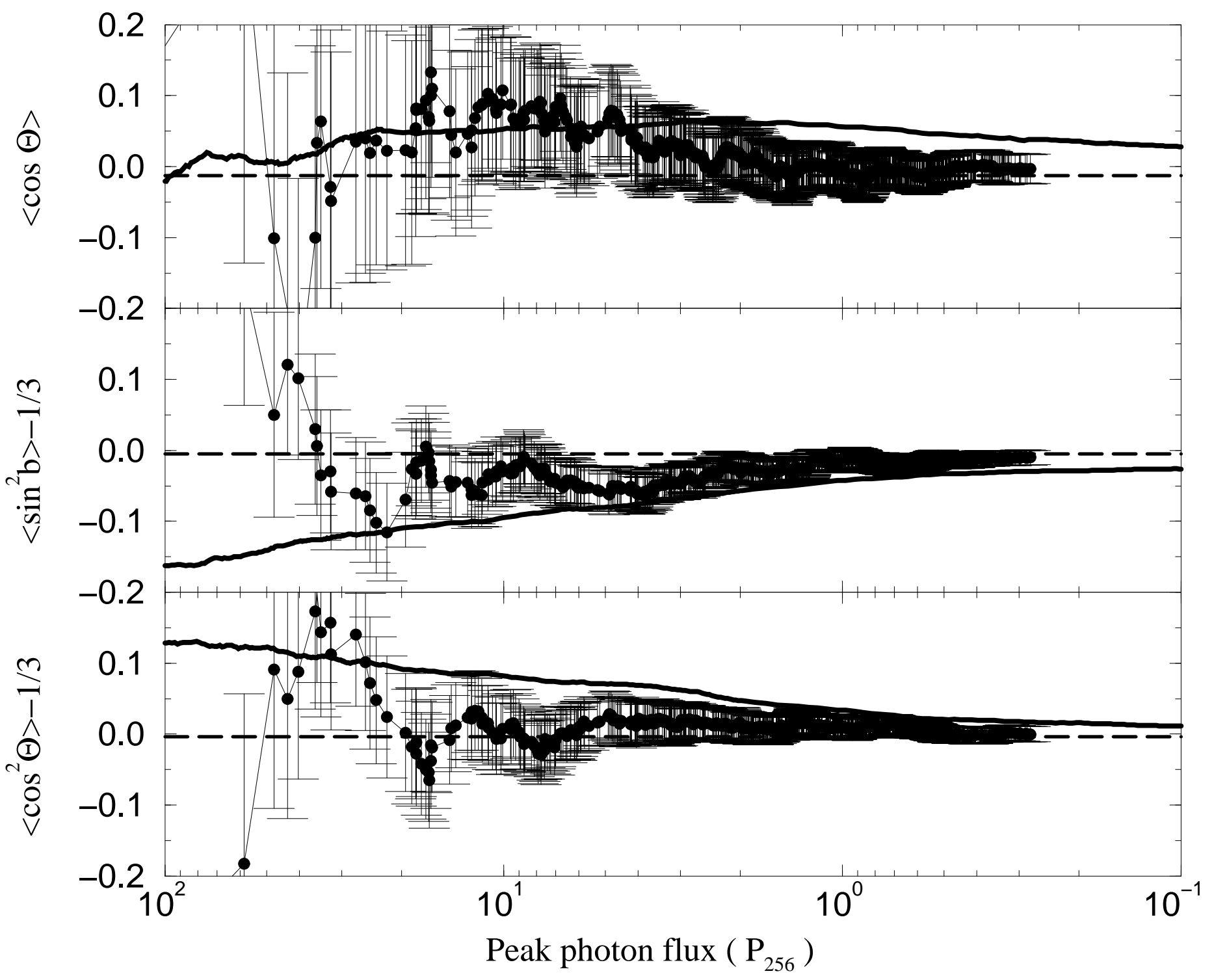

